\title{
Cranial ontogenetic variation in early saurischians and the role of heterochrony in the diversification of predatory dinosaurs
}

Christian Foth, Brandon P Hedrick, Martin D Ezcurra

Non-avian saurischian skulls underwent at least 165 million years of evolution and shapes varied from elongated skulls, such as in the theropod Coelophysis, to short and boxshaped skulls, such as in the sauropod Camarasaurus. A number of factors have long been considered to drive skull shape, including phylogeny, dietary preferences and functional constraints. However, heterochrony is increasingly being recognized as an important factor in dinosaur evolution. In order to quantitatively analyse the impact of heterochrony on saurischian skull shape, we analysed five ontogenetic trajectories using two-dimensional geometric morphometrics in a phylogenetic framework. This allowed for the comparative investigation of main ontogenetic shape changes and the evaluation of how heterochrony affected skull shape through both ontogenetic and phylogenetic trajectories. Using principal component analyses and multivariate regressions, it was possible to quantify different ontogenetic trajectories and evaluate them for evidence of heterochronic events allowing testing of previous hypotheses on cranial heterochrony in saurischians. We found that the skull shape of the hypothetical ancestor of Saurischia likely led to basal Sauropodomorpha through paedomorphosis, and to basal Theropoda mainly through peramorphosis. Paedomorphosis then led from Orionides to Avetheropoda, indicating that the paedomorphic trend previously found in advanced coelurosaurs may extend back into the early evolution of Avetheropoda. Not only are changes in saurischian skull shape complex due to the large number of factors that affected it, but heterochrony itself is complex, with a number of possible reversals throughout non-avian saurischian evolution. In general, the sampling of complete ontogenetic trajectories including early juveniles is considerably lower than the sampling of single adult or subadult individuals, which is a major impediment to the study of heterochrony on non-avian dinosaurs. Thus, the current work represents an exploratory analysis. To better understand the cranial ontogeny and the impact of heterochrony on skull evolution in saurischians, the data set that we present here must be expanded and complemented with further sampling from future fossil discoveries, especially of juvenile individuals. 
1 Cranial ontogenetic variation in early saurischians and the role of heterochrony in the

2 diversification of predatory dinosaurs

3

4 Christian Foth ${ }^{1,2,3}$, Brandon P. Hedrick ${ }^{4,5}$, Martín D. Ezcurra ${ }^{2,6,7}$

5

$6 \quad{ }^{1}$ SNBS, Bayerische Staatssammlung für Paläontologie und Geologie, Richard Wagner-Str. 10, 7 D-80333 München

$8{ }^{2}$ Department of Earth and Environmental Sciences, Ludwig-Maximilians-Universität, Richard-

9 Wagner-Str. 10, D-80333 München, Germany

$10{ }^{3}$ Department of Geosciences, University of Fribourg/Freiburg, Chemin du Musée 6, 1700

11 Fribourg, Switzerland

$12{ }^{4}$ Department of Earth and Environmental Science, University of Pennsylvania, 251 Hayden

13 Hall, 240 S 33rd Street, Philadelphia, PA 19104, USA

$14{ }^{5}$ Department of Biology, University of Massachusetts, 321 Morrill Science Center, 611 North

15 Pleasant Street, Amherst, MA 01003, USA

$16{ }^{6}$ School of Geography, Earth and Environmental Sciences, University of Birmingham,

17 Edgbaston, Birmingham B15 2TT, UK

$18{ }^{7}$ Sección Paleontología de Vertebrados, Museo Argentino de Ciencias Naturales "Bernardino

19 Rivadavia", Buenos Aires C1405DJR, Argentina

21 Correspondence: Christian Foth, Department of Geosciences, University of Fribourg/Freiburg,

22 Chemin du Musée 6, 1700 Fribourg, Switzerland

23 Tel.: +41263008944 
e-mail: christian.foth@gmx.net

25

26 Short title: Heterochrony in early saurischian skulls

27

\section{Abstract}

Non-avian saurischian skulls underwent at least 165 million years of evolution and shapes varied from elongated skulls, such as in the theropod Coelophysis, to short and box-shaped skulls, such as in the sauropod Camarasaurus. A number of factors have long been considered to drive skull shape, including phylogeny, dietary preferences and functional constraints. However, heterochrony is increasingly being recognized as an important factor in dinosaur evolution. In order to quantitatively analyse the impact of heterochrony on saurischian skull shape, we analysed five ontogenetic trajectories using two-dimensional geometric morphometrics in a phylogenetic framework. This allowed for the comparative investigation of main ontogenetic

37 shape changes and the evaluation of how heterochrony affected skull shape through both ontogenetic and phylogenetic trajectories. Using principal component analyses and multivariate regressions, it was possible to quantify different ontogenetic trajectories and evaluate them for evidence of heterochronic events allowing testing of previous hypotheses on cranial

41 heterochrony in saurischians. We found that the skull shape of the hypothetical ancestor of

42 Saurischia likely led to basal Sauropodomorpha through paedomorphosis, and to basal

43 Theropoda mainly through peramorphosis. Paedomorphosis then led from Orionides to

44 Avetheropoda, indicating that the paedomorphic trend previously found in advanced coelurosaurs may extend back into the early evolution of Avetheropoda. Not only are changes in saurischian skull shape complex due to the large number of factors that affected it, but 
47 heterochrony itself is complex, with a number of possible reversals throughout non-avian saurischian evolution. In general, the sampling of complete ontogenetic trajectories including early juveniles is considerably lower than the sampling of single adult or subadult individuals, which is a major impediment to the study of heterochrony on non-avian dinosaurs. Thus, the current work represents an exploratory analysis. To better understand the cranial ontogeny and the impact of heterochrony on skull evolution in saurischians, the data set that we present here must be expanded and complemented with further sampling from future fossil discoveries, especially of juvenile individuals.

\section{Introduction}

In an evolutionary context, heterochrony describes phenotypic changes due to shifts in the timing or rate of developmental processes in an organism relative to its ancestor, and can lead to significant evolutionary changes in body plans within relatively short periods of time (Gould, 1977; Alberch et al., 1979; McNamara, 1982; Reilly, Wiley \& Meinhardt, 1997; Klingenberg, 1998; McNamara \& McKinney, 2005). Two major types of heterochronic processes are discerned: paedomorphosis and peramorphosis. Paedomorphosis occurs when the later ontogenetic stages of an organism retain characteristics from earlier ontogenetic stages of its ancestor due to a truncation of the growth period (progenesis), decrease of the growth rate (neoteny) or a delayed onset of developmental processes (postdisplacement). In contrast, a peramorphic organism is ontogenetically more developed than the later ontogenetic stages of its ancestor due to the extension of growth period (hypermorphosis), the increase of the growth rate (acceleration) or the earlier onset of developmental processes (predisplacement) (see Gould, 1977; Alberch et al., 1979; Klingenberg, 1998). In practice, evidence for heterochronic events in 
70 evolution can be detected by comparing the ontogenetic trajectories of different taxa under the

71 consideration of their phylogenetic interrelationships (Alberch et al., 1979; Fink, 1982). Thus,

72 the concept of heterochrony connects two main fields of biological sciences: developmental and

73 evolutionary biology (Gould, 1977; Raff, 1996). When studying heterochrony, ontogenetic

74 trajectories are characterized by three separate vectors (size, shape, and ontogenetic age), which

75 allows for quantification of heterochronic processes with slope, length and position within a

76 Euclidean space (Alberch et al., 1979). In this context, geometric morphometrics is a useful

77 method for characterizing shape and size vectors to investigate heterochrony in organisms within

78 a multivariate framework (Mitteroecker, Gunz \& Bookstein, 2005).

80 Documentation of heterochrony in the vertebrate fossil record is limited. Preserved fossil

81 ontogenetic series covering the whole postnatal development of fossil species are rare due to the

82 fact that early juvenile specimens are often either lacking or incomplete. Furthermore, exact ages

83 of single ontogenetic stages are often not available, resulting in the temporal component often

84 being replaced by size, which is not an ideal variable for age (Klingenberg, 1998; Gould, 2000).

85 Nevertheless, the role of heterochrony has been recognized and discussed for the evolution of

86 multiple fossil lineages that do preserve ontogenetic series (Balanoff \& Rowe, 2007; Gerber,

87 Neige \& Eble, 2007; Schoch, 2009, 2010, 2014; Bhullar, 2012; Forasiepi \& Sánchez-Villagra,

88 2014; Ezcurra \& Butler, 2015), including non-avian dinosaurs (e.g. Long \& McNamara, 1997;

89 Erickson et al., 2004; Guenther, 2009; Bhullar et al., 2012; Canale et al., 2014). For example,

90 Long \& McNamara (1997), Erickson et al. (2004) and Canale et al. (2014) hypothesized that the

91 evolution of large body size in carcharodontosaurids and tyrannosaurids from medium-sized

92 ancestors was the result of peramorphosis. 
94 There has recently been an increasing interest in shape diversity in non-avian dinosaurs, in which

95 geometric morphometric methods have been applied on a regular basis (e.g. Bonnan, 2004;

96 Chinnery, 2004; Campione \& Evans, 2011; Hedrick \& Dodson, 2013; Lautenschlager, 2014;

97 Schwarz-Wings \& Böhm, 2014; Maiorino et al., 2015). Skull shape diversity in saurischian

98 dinosaurs has been studied in particular detail (e.g. Henderson, 2002; Young \& Larvan, 2010;

99 Rauhut et al., 2011; Brusatte et al., 2012; Bhullar et al., 2012; Foth \& Rauhut, 2013a,b), but

100 usually in relation to functional constraints, dietary preferences, phylogenetic interrelationships,

101 and macroevolutionary patterns. For example, these studies have shown that skull shape in

102 sauropodomorphs and theropods is phylogenetic constrained (Young \& Larvan, 2010; Brusatte et

103 al., 2012; Foth \& Rauhut, 2013a) and that the shape of the orbit in theropods is functionally

104 constrained (Henderson, 2002; Foth \& Rauhut, 2013a). Thus, geometric morphometrics is a

105 powerful method to quantify both intraspecific (e.g. ontogeny, sexual dimorphism,

106 polymorphism) and interspecific (e.g. systematics, macroevolution) shape variation on the basis

107 of homologous landmarks or outlines, which capture more information about shape than

108 traditional morphometric measurements (Corti, 1993; Rohlf \& Marcus, 1993; Adams, Rohlf \&

109 Slice, 2004, 2013; Slice, 2007; Mitteroecker \& Gunz, 2009; Zelditch, Swiderski \& Sheets, 2012).

110 As a result, geometric morphometrics has also been successfully applied to the study of

111 heterochrony among various tetrapod groups, in which the univariate mathematical approach of

112 Alberch et al. (1979) was adapted to a multivariate framework (e.g. Berge \& Pennin, 2004;

113 Mitteroecker et al., 2004; Mitteroecker, Gunz \& Bookstein, 2005; Liebermann et al., 2007;

114 Drake, 2011; Piras et al., 2011; Bhullar et al., 2012). However, only Bhullar et al. (2012) have

115 examined cranial shape diversity of theropod dinosaurs using multivariate methods in the context 
116 of heterochrony. This pioneering study demonstrated that recent birds have highly paedomorphic

117 skulls compared to non-avian theropods and Mesozoic birds (e.g. Archaeopteryx and

118 Enantiornithes), which evolved in a multistep transformation within the clade Eumaniraptora.

119 Furthermore, Bhullar et al. (2012) found evidence for independent peramorphic trends in the

120 skull shape of large-bodied tyrannosaurids, dromaeosaurids and troodontids and proposed a

121 similar trend for allosaurids. Finally, Bhullar et al. (2012) hypothesized a possible

122 paedomorphosis for Eoraptor and basal sauropodomorphs.

123

124 The aim of the current study is to investigate the cranial shape diversity of saurischian dinosaurs

125 by comparing the ontogenetic trajectories of different taxa from both qualitative and quantitative

126 data, using two-dimensional geometric morphometrics (2D GM). This study expands on the

127 work of Bhullar et al. (2012) who focused primarily on trends within Maniraptora, derived non-

128 avian theropods and basal avian theropods. We have built upon their study by including an

129 improved sample of basal saurischians and theropods (including a number of different

130 ontogenetic series), which should be more sensitive for testing of the heterochronic changes for

131 allosaurids and basal sauropodomorphs proposed, but not verified statistically, by Bhullar et al.

132 (2012). The phylogenetic relationships of the ontogenetic series sampled in this study are

133 integrated into an ancestor-descendant framework to look for further potential heterochronic

134 processes in the cranial evolution of saurischians. However, due to the limited number of

135 ontogenetic series known for sauropodomorphs, the current study focuses primarily on the early

136 evolution of theropods. Nevertheless, due to the limited number of ontogenetic series currently

137 available in our taxonomic sample, this work must be viewed as an exploratory study, which will

138 need to be expanded and complemented with further sampling from future fossil discoveries. 
140 Materials and Methods

\section{Institutional Abbreviations}

142 BMMS, Bürgermeister Müller Museum Solnhofen, Solnhofen, Germany; CM, Carnegie

143 Museum of Natural History, Pittsburgh, USA; GR, Ruth Hall Museum, Ghost Ranch, USA;

144 IVPP, Institute of Vertebrate Paleontology and Paleoanthropology, Beijing, China, MCZ,

145 Museum of Comparative Zoology, Harvard University, USA.

147 Taxon sampling

148 We sampled the crania of 35 saurischian dinosaur taxa (10 sauropodomorphs and 25 non-

149 pennaraptoran theropods, see Table S3 in the Supplementary Information) on the basis of

150 published reconstructions of adult (or advanced subadult) individuals in lateral view (with

151 exception of the reconstructions of the basal tyrannosauroid Dilong [IVPP V14243] and the basal

152 alvarezsauroid Haplocheirus [IVPP V15988], which were based on our personal observations).

153 The data set shows an overlap of 15 terminal taxa with that of Bhullar et al. (2012) and builds on

154 that study with an addition of 20 new taxa. Theropods with large nasal crests (e.g. Ceratosaurus,

155 Dilophosaurus, Guanlong) were excluded from the primary data set as they were found to have a

156 strong impact on the ancestral shape reconstruction (see below) of Averostra, Avetheropoda,

157 Coelurosauria and Tyrannosauroidea (see Fig. S5, Table S6 in the Supplementary Information).

158 Although cranial crests are a common structure among theropod dinosaurs (Molnar, 2005),

159 reconstruction of moderately to strongly crested hypothetical ancestors within this study would

160 necessarily be artificial due to the lack of intermediate crested forms and relatively small sample

161 size of the available data set. Only Monolophosaurus was included in the main data set because 
162 it possesses a rather moderately sized and simple nasal crest. 'Syntarsus' kayentakatae, which is

163 often reconstructed with a pair of prominent nasal crests (Rowe, 1989; Tykoski, 1998), was

164 analysed in this study without crests since this structure is probably artificial due to post-mortem

165 displacement of the nasals (Ezcurra \& Novas, 2005, 2007). As cranial crests usually represent

166 external visual signal structures (Sampson, 1999; Padian \& Horner, 2011; Hone, Naish \&

167 Cuthill, 2012), their evolutionary development most likely represents either an evolutionary

168 novelty or was sourced from regional peramorphic processes if the primordia were already

169 present in the ancestor (see discussion on the evolution of horns and frills in Ceratopsia by Long

$170 \&$ McNamara 1997). However, we generated a second data set that includes crested taxa for

171 comparison with the main data set (see below).

172

173 In our sample, five taxa preserve early ontogenetic stages allowing the capture of both juvenile

174 and adult skull shapes, which were used to reconstruct five simplified ontogenetic series,

175 containing two stages (i.e. an early juvenile and adult stage). This sample includes the basal

176 sauropodomorph Massospondylus, the basal theropod Coelophysis, the megalosaurid

177 Dubreuillosaurus, the allosauroid Allosaurus, and the tyrannosaurid Tarbosaurus (see Table S4

178 in the Supplementary Information). Two of the ontogenetic series sampled (Coelophysis and

179 Tyrannosaurus/Tarbosaurus) overlap with the data set from Bhullar et al. (2012), but we expand

180 on the previous study by including three more basal trajectories in order to concentrate on a

181 different part of the theropod tree. As the fossil record of juvenile dinosaur specimens with

182 complete skull material is rare, the number of ontogenetic series is limited. To improve

183 sampling, previous studies have included reconstructions from multiple partial juvenile skulls or

184 juveniles from closely related taxa (e.g. Bhullar et al., 2012). We implemented this approach in 
185 two cases: the reconstruction of the juvenile Coelophysis sample was based on three incomplete,

186 somewhat taphonomically deformed individuals (MCZ 4326; GR 392; CM 31375); and the

187 holotype of Sciurumimus (BMMS BK 11) was used as the juvenile representative of the

188 megalosaurid Dubreuillosaurus based on the phylogenetic analyses of Rauhut et al. (2012). In

189 contrast to Bhullar et al. (2012), we did not include the ontogenetic series of Byronosaurus,

190 Therizinosauridae (represented by a therizinosaurid embryo and the skull of Erlikosaurus) and

191 Compsognathus (with the juvenile specimen represented by Scipionyx) in the data set because

192 the postorbital region of the juvenile skulls of the former two taxa is crushed or incomplete

193 (Bever \& Norell, 2009; Kundrát et al., 2009), and the taxonomic referral of Scipionyx to the

194 clade Compsognathidae (see Dal Sasso \& Maganuco, 2011) is uncertain and maybe an artefact

195 of coding juvenile character states (see Rauhut et al., 2012).

196

\section{Two-dimensional Geometric Morphometrics (2D GM)}

198 We used 20 landmarks (LMs) and 51 semi-landmarks (semi-LMs) on our sample in order to

199 accurately capture skull shape. The landmarks were collected using the software tpsDig2 (Rohlf,

200 2005) and were classified as either type 1 (points where two bone sutures meet) or type 2 (points

201 of maximum curvature and extremities) (Bookstein, 1991) (see Fig. S1, Table S1 in the

202 Supplementary Information for full description). Type 3 landmarks (points constructed between

203 two homologous landmarks, which mainly define the shape of the skull or skull openings rather

204 than the position of exact homologous points) were not used in our study. Semi-landmarks were

205 used to capture the shape of skull openings and the skull outline by defining a number of points

206 that are placed equidistantly along respective curves (Bookstein, 1991; Bookstein et al., 1999).

207 The percent error for digitizing each landmark and semi-landmark was estimated for the skull 
208 reconstruction of the juvenile Coelophysis (with $\mathrm{n}=10$ replications) using the method described

209 by Singleton (2002). Landmark and semi-landmark error varies between 0.117 percent (LM 51 -

210 most posterior point of the descending process of the maxilla contacting the nasal and/or the

211 lacrimal) and 0.738 percent (LM 3 - contact between the maxilla and jugal along the ventral

212 margin of the skull) with a mean of 0.283 percent. The error has no significant effect on the

213 shape analyses (see Table S2 in the Supplementary Information).

215 The shape coordinates were then imported into the software package MorphoJ 1.05d

216 (Klingenberg, 2011) and superimposed using generalized Procrustes analysis (GPA). GPA

217 rotates, translates and resizes landmark coordinates of all specimens accounting for all non-shape

218 related differences between landmark configurations, leaving only shape information (Gower,

219 1975; Rohlf \& Slice, 1990). Although semi-landmarks have fewer degrees of freedom than

220 regular landmarks (and thus contain less shape information) (Bookstein, 1991), we treated

221 landmarks and semi-landmarks as equivalent for GPA (Zelditch, Swiderski \& Sheets, 2012) and

222 did not slide the semi-landmarks. The sliding process created considerable artificial deformation

223 on the Procrustes-fitted shape in some taxa (see Fig. S2 in the Supplementary Information).

224 However, due to the equivalent weighting of landmarks and semi-landmarks, it should be kept in

225 mind that the shape information captured by the semi-landmarks strongly influences the results

226 (Zelditch, Swiderski \& Sheets, 2012; see below). In order to estimate the influence of the semi-

227 landmarks on the shape data, all analyses described below were also applied to an additional data

228 set that included only landmark data (see Supplementary Information). 
230 The generated Procrustes coordinates were used to compare juvenile and adult skull shapes to

231 each other in each ontogenetic series to find ontogenetic patterns between and within taxa.

232 Furthermore, the Procrustes coordinates of all taxa (including juvenile specimens) were

233 subjected to an exploratory principal components analysis (PCA) using the covariance matrix

234 generated from Procrustes coordinates. PCA simplifies descriptions of variation among

235 individuals by creating new sets of variables that are linear combinations of the original set such

236 that the new sets are independent from one another and have zero covariance. The principal

237 components (PCs) describe successively smaller amounts of total variance of the sample. This

238 allows for a larger proportion of the variance to be described using a smaller number of variables

239 than the original data would have allowed (Zelditch, Swiderski \& Sheets, 2012). A multivariate

240 regression of the Procrustes coordinates against log-transformed centroid sizes (= square root of

241 the sum of the squared distances of each landmark to the centroid of the landmark configuration,

242 Zelditch, Swiderski \& Sheets, 2012) was performed to test if the skull shape variation is

243 correlated with size and contains allometric information (Drake \& Klingenberg, 2008).

\section{Quantification of ontogenetic trajectories}

246 The different ontogenetic trajectories generated in the PCA and regression analyses were

247 compared to each other by calculating pairwise two-dimensional angles between different

248 trajectories based on the PC values of the first three axes, which are the significant principal

249 components (significance calculated using the broken stick method, see Jackson, 1993). Each of

250 the two-stage ontogenetic trajectories was described as a phenotypic change vector, $\Delta \vec{y}_{i}=\vec{y}_{i j}-\vec{y}_{i k}$

251 , with two shape traits (PC 1 vs. PC 2 and PC 1 vs. PC 3), where $i$ is a specific ontogeny between

252 two fixed stages, juvenile $(j)$ and adult ( $k$ ) (Collyer \& Adam, 2007). The difference in direction 
253 (angle) between the ontogenetic phenotypic change vectors $\Delta \vec{y}_{a}, \Delta \vec{y}_{b}$ was calculated using the

254 dot product $\cos ^{-1}\left(\Delta \vec{y}_{a}, \Delta \vec{y}_{b}\right)=\frac{\Delta \vec{y}_{a} \cdot \Delta \vec{y}_{b}}{\left|\Delta \vec{y}_{a}\right|\left|\Delta \vec{y}_{b}\right|}$. PC values were employed to calculate the length

255 of each ontogenetic trajectory. Lengths and angles were used to characterise the differences

256 between the ontogenetic trajectories in relation to shape variation.

258 Phylogenetic framework for heterochronic analyses

259 In an evolutionary context, heterochrony is defined as the change in the timing or rate of

260 developmental processes in ancestor-descendant relationships (Alberch et al., 1979; Fink, 1982;

261 Klingenberg, 1998), and thus a direct comparison of ontogenetic trajectories from different

262 species (as terminal taxa) can be problematic because it is hard to determine which trajectory

263 would represent the ancestral and the descendant form, respectively (see Fink 1982). This is

264 exacerbated when the supposed ancestral (terminal) species possesses an unknown, long

265 evolutionary history resulting from a ghost lineage. This problem can be partially solved using a

266 phylogenetic approach (see Alberch et al., 1979; Fink 1982; Balanoff \& Rowe. 2007; Bhullar,

267 2012; Fritsch, Bininda-Emonds \& Richter, 2013; Mallon et al., 2015), in which the ancestor of

268 two sister (terminal) taxa is represented by the hypothetical last common ancestor (Hennig,

269 1966). Therefore, on the basis of the phylogenetic distribution of the five ontogenetic series

270 sampled we calculated hypothetical ancestral ontogenetic trajectories for Saurischia,

271 Neotheropoda, Orionides and Avetheropoda using ancestral shape reconstructions as follows

272 (see Fig. S3, S4 in the Supplementary Information). An informal supertree (sensu Butler \&

273 Goswami, 2008) including all taxa with adult individuals was created based on recent

274 phylogenetic analyses (see Fig. S3, S4 in the Supplementary Information): basal 
275 Sauropodomorpha (Cabreira et al., 2011), Coelophysoidea (Ezcurra \& Novas, 2007),

276 Ceratosauria (Pol \& Rauhut, 2012), Tetanurae (Carrano, Benson \& Sampson, 2012), and

277 Coelurosauria (Turner et al., 2012; Loewen et al., 2013). The phylogenetic position of Eoraptor

278 follows Martínez et al. (2011) and Martínez, Apaldetti \& Abelin (2013). The position of

279 Adeopapposaurus as sister taxon of Massospondylus follows Martínez (2009). The position of

280 Herrerasaurus and Tawa at the base of Theropoda is based on Sues et al. (2011). Zupaysaurus

281 was placed outside Coelophysoidea as one of the successive sister taxa of Averostra (Smith et

282 al., 2007; Sues et al., 2011; Ezcurra, 2012). The supertree was time-calibrated using the

283 stratigraphic age of each taxon (as mean of time interval) (see Table S3, S5 in the Supplementary

284 Information). The assignment of branch lengths was performed in R ( R Development Core

285 Team, 2011) using the APE package (version 2.7-2; Paradis, Claude \& Strimmer, 2004) and a

286 protocol written by Graeme Lloyd (see http://www.graemetlloyd.com/methdpf.html) for

287 adjusting zero branch lengths by sharing out the time equally between branches (see Brusatte $e t$ 288 al., 2008; Brusatte, 2011), and adding an arbitrary length of 1 million years to the root. The time-

289 calibrated supertree was imported into the software package Mesquite 2.72 (Maddison \&

290 Maddison, 2009). Subsequently, Procrustes coordinates and centroid sizes of the adult

291 representatives of the taxa were mapped onto the supertree as continuous characters using square

292 change parsimony. This algorithm performs an ancestral state reconstruction by collating the

293 sum of squared changes of continuous characters along all branches of a tree and estimates the

294 most parsimonious ancestral states by minimizing the total sum of squared changes across the

295 tree (Maddison, 1991). In the next step we tested if the continuous data contains a phylogenetic

296 signal. We performed a permutation test in MorphoJ in which the topology was held constant

297 and both the Procrustes-fitted shape data and the centroid size for each taxon were randomly 
298 permuted for all the terminals across the tree 10,000 times (Laurin, 2004; Klingenberg \&

299 Gidaszewski, 2010). The data are considered to contain a statistically significant phylogenetic

300 signal if the squared length of the original supertree occurs in at least $95 \%$ of the randomly

301 generated trees. Additionally, we quantified phylogenetic signal in our data using a multivariate

302 form of the $K$ statistic with 10,000 replications (Blomberg et al., 2003; Paradis, 2012; Adams,

303 2014) in R using the package geomorph (Adams \& Otárola-Castillo, 2013). This test estimates

304 the strength of a phylogenetic signal in a data set in relation to a simulated Brownian motion

305 model, which is expressed as $K$ and $p$ values.

306

307 To obtain ancestral ontogenetic trajectories, the protocol described above was repeated in a new

308 nexus file containing the Procrustes-fitted shapes and centroid sizes of the juvenile specimens.

309 As the juvenile data set is only represented by five taxa, the original supertree was pruned such

310 that only these taxa remained, retaining the original time-calibration. Finally, the ancestral

311 Procrustes-fitted shapes and centroid sizes of both juvenile and adult Saurischia, Neotheropoda,

312 Orionides and Avetheropoda were exported and combined with the respective data from the

313 ontogenetic trajectories of the terminal taxa. The ancestral Procrustes-fitted shape of Averostra

314 was not considered because no ceratosaur juveniles have been published in detail so far (see

315 Madsen \& Welles, 2000). The new data set including the five terminal and four ancestral

316 ontogenetic trajectories was loaded again into MorphoJ.

\section{Regression analyses of ontogenetic trajectories}

319 A multivariate, pooled within-group regression of shape against log-transformed centroid size

320 including terminal taxa and hypothetical ancestors (see above) was performed (Piras et al., 2011; 
321 Bhullar et al., 2012; Zelditch, Swiderski \& Sheets, 2012), in which the Procrustes coordinates

322 were transformed into a regression score (see Drake \& Klingenberg, 2008). In contrast to many

323 previous studies of heterochrony using geometric morphometrics, which compare only the

324 ontogenetic trajectories of terminal taxa, our approach allows the determination of possible

325 heterochronic patterns between ancestors and descendants. The different ontogenetic trajectories

326 were compared regarding slope, length, angles and range of shape variation spanned by the

327 predicted regression score. The angles between ontogenetic trajectories were calculated based on

328 Procrustes distances and centroid sizes (see above).

329

330 As mentioned above, studies of heterochrony require size, shape and ontogenetic age as

331 independent vectors (Klingenberg, 1998). Due to missing data on the individual age of the

332 specimens, ontogenetic age could not be taken into account. As a consequence, the regression

333 analysis explores allometry and not heterochrony (Klingenberg \& Spence, 1993; Klingenberg,

334 1998; Gould, 2000). While some heterochronic processes can result from allometric changes

335 (e.g. acceleration and neoteny), allometric studies allow only conclusions regarding

336 paedomorphosis and peramorphosis (Klingenberg \& Spence, 1993; Klingenberg, 1998), which

337 are expressed by the shape vector (i.e. regression score). Peramorphosis can be inferred if the

338 adult individual of the descendant trajectory falls along higher regression scores than the

339 respective ancestral one, whereas paedomorphosis can be inferred based along lower scores. To

340 verify the results of such regression analyses we repeated the analysis using Euclidean distance,

341 which is equivalent to Procrustes distance (see Singleton, 2002; Tallman et al., 2013) as a

342 separate shape vector measuring differences in shape. The Euclidean distance matrix was

343 calculated in PAST 3.05 (Hammer, Harper \& Ryan, 2001) on the basis of the Procrustes 
344 coordinates of terminal taxa and hypothetical ancestors (see above), which were exported from

345 MorphoJ. For regression analysis, the juvenile specimen of Massospondylus, which represents

346 the sample with the smallest centroid size, was set to zero for aligning the distance values of the

347 remaining taxa (Fig. 4).

348

349 To test if the shape changes, and as a result the presence of heterochrony, of an ancestor-

350 descendant relationship are statistically meaningful, we calculated the confidence interval (CI) of

351 the differences between regression scores and Euclidean distances of terminal and ancestral taxa

$352(n=68)$ and compared them with the differences of ancestral and descendant regression scores

353 from the sub-sample containing the ontogenetic trajectories. Changes were considered significant

354 if the differences between regression scores were at least 1.5 times higher than the CI value (see

355 Cumming, Fidler \& Vaux, 2007).

356

357 For comparison, we performed another PCA with the data set containing just terminal and

358 ancestral ontogenetic trajectories and calculated the angles and lengths of the trajectories on the

359 basis of the first two principal components, which were found to contain all significant shape

360 information based on the broken stick method (see above).

361

362 Finally, the ancestral shape reconstructions calculated for the adult representatives of the taxa

363 were used to qualitatively discuss the evolutionary changes within basal Sauropodomorpha and

364 Theropoda with respect to the ontogenetic changes and heterochronic trends found in the 365 different trajectories. 


\section{Results}

\section{General ontogenetic changes}

369 The juveniles of the sauropodomorph Massospondylus and the theropods that were sampled here

370 tend to have skulls with a short and abruptly tapering snout, short antorbital fenestrae, large

371 subcircular orbits, slender jugals, and dorsoventrally deep orbital and postorbital regions relative

372 to the snout. In addition, the jaw joint is more anteriorly placed relative to the occiput, with

373 exception of the juvenile specimen of Allosaurus sampled here. The general ontogenetic pattern

374 includes an elongated and dorsoventrally deeper snout relative to the orbital and postorbital

375 regions, and also a relative increase in size of the antorbital fenestra, which correlates with a

376 relative decrease in size of the orbit. Finally, the jugal becomes more massive in all taxa, which

377 is more pronounced in the large-bodied theropods Allosaurus and Tarbosaurus (Fig. 1). The

378 relative elongation of the snout and antorbital fenestra were not observed in the Allosaurus or

379 Tarbosaurus ontogenies, which is probably due to the fact that the juveniles sampled do not

380 represent the earliest ontogenetic stages (Loewen, 2009; Tsuihiji et al., 2011, see discussion).

381 However, the discovery of an isolated maxilla identified as a hatchling allosauroid might indicate

382 that the snout of early Allosaurus juveniles was probably short and subsequently increased in

383 relative length during early ontogeny (Rauhut \& Fechner, 2005).

385 In addition to these more general ontogenetic modifications, individual taxa show specific shape 386 changes (Fig. 1):

a) In Massospondylus the external naris becomes larger and expands dorsally. The relative size. The jaw joint moves anteroventrally. 
390 b) In Coelophysis the external naris becomes smaller and shifts anteriorly. The notch of the

391 alveolar margin between the premaxilla and maxilla decreases in relative size during

392 ontogeny, while the alveolar margin of the premaxilla becomes more aligned with that of

393 the maxilla. The descending process of the lacrimal becomes more slender

394 anteroposteriorly. The postorbital becomes more gracile in its relative shape. The

395 infratemporal fenestra increases in relative size. The jaw joint moves posterodorsally.

396 c) In the megalosaurid taxon, the external naris becomes relatively larger and expands

397 posteriorly. The lacrimal is inclined strongly backwards and the postorbital becomes

398 relatively more robust. The infratemporal fenestra increases in its relative size. The jaw

399 joint moves posteriorly.

400 d) In Allosaurus the external naris does not change in relative size, but shifts ventrally. The

401 descending process of the lacrimal becomes more massive anteroposteriorly. The

402 lacrimal develops a prominent dorsal horn through ontogeny. In contrast to previous taxa,

403 the postorbital region of Allosaurus increases dorsoventrally such that the postorbital,

404 quadratojugal and squamosal become relatively more robust. The ventral shift of the

405 jugal leads to the formation of a wide angle between the ventral margins of the maxilla

406 and jugal. Due to its posteroventral expansion, the postorbital affects the shape of the

407 infratemporal fenestra. However, the infratemporal fenestra does not decrease in its

408 relative size, but shifts anteroventrally. The jaw joint moves anteroventrally.

409 e) In Tarbosaurus the external naris does not change in relative size, but shifts dorsally. As

410 in Allosaurus, the descending process of the lacrimal becomes more massive. The same is

411 true for the postorbital region, which increases in depth dorsoventrally. This change is 

squamosal. The jaw joint moves posteroventrally.

\section{Principal component analysis and phylogenetic correlation}

416 The first three principal components account for $68.0 \%$ of the total variation (PC 1: $30.8 \%$; PC

417 2: $23.9 \%$; PC 3: $13.3 \%$ ), in which PC 2 and PC 3 contain the main allometric shape information 418 (see Table S12 in the Supplementary Information). PC 1 describes the overall skull depth, size 419 and anteroposterior position of the external naris, length of the premaxilla, size of the maxillary

420 antorbital fossa, and position of the lacrimal and postorbital on the anteroposterior axis (affecting

421 the size of the antorbital fenestra, orbit and infratemporal fenestra). The dorsoventral dimension

422 of the orbit is affected by the relative depth of the entire orbital and postorbital regions, while

423 that of the infratemporal fenestra is affected by the relative position of the jugal-quadratojugal

424 bar. The variation in the depth of the skull also affects the position of the jaw joint on the 425 dorsoventral axis (Fig. 2c). PC 2 describes the length of the snout caused by variation in the 426 length of the maxilla and inclination and anteroposterior position of the lacrimal. The inclination

427 of the lacrimal affects the size of the antorbital fenestra, while both position and inclination 428 affect the anteroposterior dimension of the orbit. PC 2 also accounts for the length and the 429 dorsoventral position of the external naris and size of the upper temporal region (Fig. 2c). PC 3

430 describes the length of the premaxilla, posterior extension of the external naris, dorsoventral

431 height of the maxilla, and anteroposterior dimension of the ventral process of the lacrimal (which

432 affects the shape of the antorbital fenestra and orbit). The shape of the orbit is further affected by

433 the anteroposterior dimension of the jugal-postorbital bar. Further variation captured by PC 3 is

434 related to the shape of the skull roof in the orbital and postorbital regions, dorsoventral height of 
435 the infratemporal fenestra, and position of the jaw joint on the anterodorsal-posteroventral axis 436 (Fig. 2c).

438 The permutation tests and the multivariate $K$ statistic recovered that both Procrustes-fitted shapes

439 (tree length weighted by branch lengths $=0.5108, p<0.0001 ; K=0.2607, p=0.0016$ ) and

440 centroid size (tree length weighted by branch lengths $=8.3598, \mathrm{p}=0.0005 ; K=0.8900, p=$

$4410.0002)$ are correlated with phylogeny. Furthermore, the multivariate regression analysis reveals

442 that skull shape is significantly correlated with centroid size (correlation index: 15.32\%, $p$

$443<0.0001)$ (Fig. 4a, Table S12 in the supplementary Information).

445 Ontogenetic trajectories in the PCA morphospace

446 Based on the PCA results of the original data set (i.e. including semi-landmarks), the ontogenetic

447 trajectories are not uniform (Fig. 2, Table 1). The trajectory of Allosaurus is short and mainly

448 explained by shape variation captured by PC 1, while that of Tarbosaurus is also short, but

449 mainly explained by PCs 1 and 3. The third principal component has stronger influence on the

450 ontogenetic shape variation in Tarbosaurus based on the length of its trajectory. Compared to

451 Allosaurus and Tarbosaurus, the other ontogenetic trajectories are longer. The trajectory of

452 Coelophysis is mainly explained by the shape variation captured by PCs 1 and 2, while its slope

453 is opposite to the direction along PC 1 compared to the trajectories of Allosaurus and

454 Tarbosaurus. Based on the angles, the ontogenetic trajectories of Massospondylus and the

455 megalosaurid taxon are mainly influenced by the shape variation captured by PCs 2 and 3, in

456 which the ontogenetic trajectory of Massospondylus is directed in the opposite direction along

457 PC 3 to that of the megalosaurid taxon and Tarbosaurus. However, the length of the trajectories 
458 indicates that the second principal component has major influence on the shape variation in both

459 species during ontogeny.

460

461 The PCA reveals that the ontogenetic elongation of the snout is primarily related to a relative

462 increase in the length of the maxilla (PCs 1,2). In Massospondylus and the megalosaurid taxon

463 the ontogenetic elongation of the snout is further affected by the relative increase of the length of

464 the premaxilla (PC 3). The relative increase in snout depth results mainly from a ventral

465 expansion of the maxilla, which is more prominent in Allosaurus and Tarbosaurus than in other

466 taxa (PCs 1, 3). In the megalosaurid taxon and Allosaurus, maxillary deepening occurs together

467 with a dorsoventral expansion of the nasal (PC 1). Additionally, dorsoventral expansion of the

468 premaxilla is observed in Allosaurus and Tarbosaurus (PC 1). The relative elongation of the

469 snout in Massospondylus, the megalosaurid taxon and Coelophysis correlates with a relative

470 increase in the anteroposterior length of the antorbital fenestra, caused by a posterior shift of the

471 lacrimal and elongation of the maxilla (PCs 1, 2). Additionally, in Coelophysis the anterior

472 border of the antorbital fenestra extends anteriorly (PC 1). In both Massospondylus and the

473 megalosaurid taxon, the antorbital fenestra is shifted posteriorly during ontogeny (PC 2). The

474 megalosaurid taxon shows a further dorsal expansion of the antorbital fenestra (PC 3), not seen

475 in the latter two taxa. Although no relative size changes could be observed in the antorbital

476 fenestrae of Allosaurus and Tarbosaurus, the antorbital fenestra of Allosaurus shifts

477 posterodorsally during ontogeny, whereas that of Tarbosaurus shifts ventrally. In most

478 trajectories, the most anterior point of the antorbital fossa shifts posteriorly during ontogeny (PCs

$4791-3)$, but a relative decrease in the length of the maxillary antorbital fossa is present in

480 Allosaurus and Tarbosaurus (PC 1). In the megalosaurid taxon, the anterior margin of the 
481 antorbital fossa shifts ventrally, whereas in Coelophysis it shifts anteriorly (PC 1), which

482 correlates with the anterior elongation of the antorbital fenestra in this taxon (see above). As

483 mentioned above, the orbit decreases in relative size in all taxa during ontogeny (PCs 1-3). In

484 Coelophysis and Massospondylus this is related to a relative shift of the lacrimal posteriorly (PCs

485 1,2). In the megalosaurid taxon, Allosaurus and Tarbosaurus the relative size reduction is

486 correlated with a change in orbital shape from subcircular to oval. In the megalosaurid taxon

487 these changes are linked to a posterior shift of the lacrimal (PC 2) and anterior shift of the

488 postorbital and ascending process of the jugal (PC 3), which is correlated with an anterior

489 extension of the infratemporal fenestra. In Allosaurus, the ontogenetic changes of the orbit are

490 related to the posterior extension of the lacrimal and anterior shift of the postorbital and

491 ascending process of the jugal (PC 1). Additionally, the orbit of Allosaurus is shifted slightly

492 dorsally. In Tarbosaurus, these changes result from an anterior extension of both the postorbital

493 and ascending process of the jugal (PC 3). The orbit of Tarbosaurus becomes posteriorly

494 constricted by an anterior shift of the ventral process of the postorbital, forming a suborbital

495 process.

496

497 We examined the differences in the trajectory directions when terminal and ancestral ontogenetic

498 series are compared to each other (Fig. 3, Table 2). The significant shape variation evaluated via

499 the broken stick method is described by the first two principal components (PC1: 50.39 \%; PC2:

$50020.79 \%$ ). Both axes are correlated with centroid size (see Table S12, Supplementary

501 Information). The ontogenetic trajectory of Coelophysis in mainly influenced by PC 1, while that

502 of the megalosaurid taxon, Massospondylus and all ancestral trajectories is influenced by both

503 PC 1 and 2, in which the first principal component is found to have a higher impact on the shape 
504 variation during ontogeny. In contrast, the ontogenetic trajectories of Tarbosaurus and

505 Allosaurus are mainly influenced by PC2.

506

507 Ontogenetic trajectories in the regression analyses

508 The ontogenetic trajectory of Massospondylus is longer than that of the hypothetical ancestor of

509 Saurischia for both shape variables (regression score and Euclidean distance), while the values of

510 the shape variables are significantly lower. However, the slope of the trajectory of

511 Massospondylus based on the regression score is less pronounced than that of the saurischian

512 ancestor, while it is more pronounced for the Euclidean distance (Fig. 4b, c, Table 3, 4). In

513 contrast, the ontogenetic trajectory of the hypothetical ancestor of Neotheropoda is slightly

514 longer and has a greater slope, while the regression score and the Euclidean distance of the adult

515 individual are significantly higher than that of the saurischian ancestor. Coelophysis possesses a

516 longer and steeper ontogenetic trajectory for both shape variables with significantly higher

517 values than the hypothetical ancestor of Neotheropoda (Fig. 4b, c, Table 3, 4). The ontogenetic

518 trajectory of the hypothetical ancestor of Orionides is shorter and has a lower slope than that of

519 the neotheropod ancestor. The regression score of the adult individual is significantly higher,

520 while the Euclidean distance is lower, but not significantly different. Compared to the

521 hypothetical ancestor of Orionides, the megalosaurid taxon has a longer and steeper ontogenetic

522 trajectory, with a significantly higher value for both shape variables (Fig. 4b, c, Table 3, 4). In

523 contrast, the ontogenetic trajectory of the hypothetical ancestor of Avetheropoda is shorter,

524 possessing a lower slope and significantly lower regression score and Euclidean distance for the

525 adult individual, when compared to the hypothetical ancestor of Orionides. The ontogenetic

526 trajectories of Allosaurus and Tarbosaurus are longer than that of the hypothetical ancestor of 
527 Avetheropoda. Both trajectories show a slope decrease compared to their common ancestor.

528 Interestingly, the slope is almost zero when the Euclidean distance is applied as shape variable,

529 indicating only minor shape changes during the ontogeny as sampled. For Allosaurus, both shape

530 values of the adult individual are higher than that of the ancestor, but only the regression score is

531 significant. In contrast, the regression score of the adult individual of Tarbosaurus is

532 significantly lower than that of the hypothetical ancestor of Avetheropoda, while the Euclidean

533 distance results in a higher, but non statistically significant value (Fig. 4b, c, Table 3, 4).

535 Based on the regression analysis, taxa with higher regressions scores tend to have elongated

536 skulls with long and slender snouts that have a rounded anterior end, and possess

537 anteroposteriorly long antorbital fenestrae, oval orbits and a post-rostrum only slightly

538 dorsoventrally higher than the snout. The maxilla increases in its relative length, but also

539 expands ventrally. The ascending process of the maxilla, the anterior and ascending processes of

540 the jugal, and postorbital become more massive. In contrast, low regression scores account for

541 skull shapes where these features are less pronounced, developed or even show opposite trends.

542 When compared to the regression analyses containing all taxa, the relative position, length and

543 slopes of the ontogenetic trajectories of the terminal taxa is almost identical (Fig. 4a), supporting

544 the robustness of the results recovered.

546 Discussion

547 Ontogenetic patterns

548 Our knowledge of the cranial ontogeny of non-avian dinosaurs remains fragmentary. Previous

549 studies on cranial ontogeny have often been based on single species (Gow, Kitching \& Raath, 
550 1990; Carr \& Williamson, 2004; Horner \& Goodwin, 2006; Hübner \& Rauhut, 2010; Campione

551 \& Evans, 2011; Mallon et al., 2011; Canale et al., 2014; Frederickson \&Tumarkin-Deratzian,

552 2014), while only a small number of studies have investigated this topic on the interspecific level

553 (Carr, 1999; Evans, 2010; Bhullar et al., 2012; Mallon et al., 2015). As is common in other

554 animal groups, closely related species often undergo similar ontogenetic changes (see Evans,

555 2010; Mallon et al., 2015), while ontogenetic trajectories become more different with increased

556 phylogenetic distance (see Bhullar et al., 2012) or in the case of a single taxon evolving extreme

557 ontogenies compared to their relatives (Horner \& Goodwin, 2009, see also Erickson et al., 2004).

558 Despite the large phylogenetic distance between the ontogenetic series sampled here, the present

559 study reveals that the cranial ontogeny of saurischian dinosaurs undergoes some general patterns,

560 including the relative elongation and dorsoventrally heightening of the preorbital region,

561 decrease in orbit size and increase in jugal robustness. However, the PCA shows that the

562 different ontogenetic trajectories differ strongly in length, direction and also the location within

563 the morphospace. Here, the theropod taxa are markedly separated from the sauropodomorph

564 Massospondylus, which is morphologically very distinct from other basal sauropod taxa. This is

565 especially obvious in the large distance within morphospace between Massospondylus and

566 Coelophysis, which represent the most basal ontogenetic series of each clade indicating a strong

567 diversification of skull shape in the early evolution of Saurischia. This may be related to

568 differentiations along both lines of Saurischia in terms of ecology, including trophic

569 specializations (see Tykoski \& Rowe, 2004; Barrett \& Rayfield, 2006; Langer et al., 2010;

570 Sakamoto, 2010). The fact that the separation within the morphospace already take place among

571 juvenile specimens indicates that these specializations might appear very early in ontogenetic 
572 development. Although the distances among such specimens in morphospace are large, the

573 trajectories show that both species still share similar trends in cranial development (Fig. 2, 3).

574

575 Although occupying a similar area of morphospace, the ontogenetic trajectory of the

576 megalosaurid taxon differs markedly from that of Allosaurus and Tarbosaurus, showing more

577 similarity with that of Massospondylus and Coelophysis, which share in common the relative

578 elongation of the snout. The latter process probably represents a morphological trend within

579 megalosaurids (Therrien \& Henderson, 2007; Sadleir et al., 2008), while large-bodied

580 allosauroids and tyrannosaurids tend to have rather deeper than long skulls (see Brusatte et al.,

581 2012; Foth \& Rauhut, 2013). However, as several medium-sized tyrannosauroids also have

582 elongated snouts (Li et al., 2010; Brusatte, Carr \& Norell, 2012; Lü et al., 2014; Porfiri et al.

583 2014), their ontogenetic trajectories would probably more closely resemble that of the

584 megalosaurid taxon. One has to take into account that the length and direction of the ontogenetic

585 trajectories of Allosaurus and Tarbosaurus are likely influenced by the fact that the juvenile

586 specimens are ontogenetically more developed compared to that of the megalosaurid taxon (see

587 below). Assuming that the hatchlings of Allosaurus and Tarbosaurus also had short, tapering

588 snouts, the trajectory would probably be more similar in length and direction to that of the

589 megalosaurid taxon.

590

\section{Heterochronic patterns}

592 Previous workers have hypothesized that skull shape diversity in theropods and

593 sauropodomorphs was driven by phylogenetic interrelationships, dietary preferences (Young \&

594 Larvan, 2010; Brusatte et al., 2012; Foth \& Rauhut, 2013a), functional constraints (Henderson, 
595 2002; Foth \& Rauhut, 2013a), but also heterochrony (Long \& McNamara, 1997; Bhullar et al.,

596 2012). This study builds on the recent heterochronic analysis of Bhullar et al. (2012), who

597 primarily examined derived non-avian theropods and basal avians on the basis of a great number

598 of ontogenetic trajectories of non-avian coelurosaurs and an extant phylogenetic bracket of

599 crocodylians and birds, covering a broader scale of archosaurian craniofacial shape variation.

600 However, by sampling and comparing ontogenetic trajectories of more basal saurischian taxa,

601 our data set allows for reevaluation of the conclusions presented by Bhullar et al. (2012) with

602 regards to basal sauropodomorphs, allosauroids and tyrannosauroids. The current study supports

603 the influence of heterochrony on the cranial evolution of some saurischian lineages. When the

604 differences of the regressions scores $(\Delta \mathrm{RS})$ and the Euclidean distances $(\Delta \mathrm{ED})$ in an ancestor-

605 descendant relationship are compared, the significant decrease of the shape values indicates

606 potential paedomorphosis for the skull shape of Massospondylus and the hypothetical ancestor of

607 Avetheropoda, while the skulls of Coelophysis, the megalosaurid taxon and the hypothetical

608 ancestor of Neotheropoda, might be peramorphic. Thus, the current analyses support a

609 paedomorphosis for basal sauropodomorphs as predicted by Bhullar et al. (2012). Due to

610 contradicting results regarding shape differences, no heterochronic pattern can be inferred for

611 Allosaurus, Tarbosaurus and the hypothetical ancestor of Orionides. Thus, the current analyses

612 do not support the predicted cranial peramorphosis for the allosauroids and tyrannosaurid lineage

613 (Long \& McNamara, 1997; Bhullar et al. 2012; Canale et al., 2014), while studies on growth

614 (Bybee, Lee \& Lamm, 2006; Erickson et al., 2006) and body size evolution (Dececchi \&

615 Larsson, 2013; Benson et al., 2014; Lee et al., 2014) in theropods indicate such a trend.

616 However, this conflict is probably caused by incomplete sampling of ontogenetic trajectories,

617 which affects the estimated shape of the hypothetical ancestor of Avetheropoda. A further 
618 expansion of the sampling of ontogenetic trajectories of saurischian taxa and the inclusion of an

619 extant phylogenetic bracket (see Bhullar et al., 2012), would probably change some aspects of

620 the analytical outcomes of this study (see below).

621

622 The increase in slopes in the ontogenetic trajectories of Neotheropoda, Coelophysis and the 623 megalosaurid taxon, when compared to their ancestors, might show evidence for peramorphic 624 acceleration. However, with a few exceptions, bone histology of basal theropods (e.g.

625 Coelophysis and Syntarsus) is not well studied, so that this cannot be confirmed by growth 626 patterns. Several studies on body size evolution support a peramorphic trend, showing an 627 increase of size from the hypothetical ancestor of Saurischia over Neotheropoda towards 628 megalosaurids (Irmis, 2011; Dececchi \& Larsson, 2013). In contrast, the relative decrease in 629 slope in the ontogenetic trajectory of the hypothetical ancestor of Avetheropoda might indicate 630 neoteny. But again this cannot be confirmed by bone histological data at this time. Dececchi \& 631 Larsson (2013) and Lee et al. (2014) found a decrease of body size from the hypothetical 632 ancestor of Tetanurae towards Avetheropoda, supporting a paedomorphic trend in body size. For 633 Massospondylus, the situation is not entirely clear, as our two shape variables led to conflicting 634 results regarding the slope, when compared with the saurischian ancestor. Thus, no underlying 635 heterochronic process can be diagnosed for the paedomorphic skull shape of Massospondylus. 636 Although basal sauropodomorphs show a gradual trend towards bigger body size (Sander et al., 637 2010; Irmis, 2011; Benson et al., 2014) and longer, accelerated growth (Chinsamy 1993;

638 Erickson, Rogers \& Yerby, 2001; Klein \& Sander 2007), skull size decreased relatively (Rauhut 639 et al., 2011). This relative shrinking might be the reason for the maintenance of a more juvenile 640 skull shape in the early evolution of sauropodomorphs. However, due to the lack of information 
641 regarding the ontogenetic age of the individuals, the deduction of heterochronic process related

642 to the slope (i.e. neoteny and acceleration) has to be considered with caution (see below).

643

644 The results of the regression analyses can be further used to interpret evolutionary shape changes

645 found between hypothetical ancestors and terminal taxa in the ancestral shape reconstruction

646 analyses of the main sample (i.e. continuous character mapping of the Procrustes-fitted shapes)

647 in terms of paedomorphic or peramorphic trends (Fig. 5). Comparing the skull shape of the

648 hypothetical ancestor of Saurischia to that of Sauropodomorpha indicates a possible initial

649 paedomorphosis in the evolution of the latter group as shown by the regression analyses, which

650 is depicted by a decrease in the relative length of the preorbital region and an increase in the

651 relative orbital size and depth of the postorbital region. As stated above, Bhullar et al. (2012)

652 already proposed a cranial paedomorphosis for basal sauropodomorphs after finding a strong

653 similarity between the skull shape of Eoraptor and the juvenile theropod Coelophysis, which had

654 been also highlighted qualitatively by previous authors (e.g. Ezcurra, 2007). In addition, Foth

655 (2013) has shown that the skull shape of Eoraptor and Pampadromaeus resembled that of the

656 juvenile theropods Sciurumimus and Juravenator. In Eusauropoda the snouts become more

657 aberrant due to a dorsal shift of the external naris, posterodorsal extension of the premaxilla,

658 elongation of the ascending process of the maxilla and modification of the postorbital region,

659 affecting the relative size of the jugal and postorbital, which become more gracile (Wilson \&

660 Sereno, 1998; Rauhut et al., 2011). While the shape changes in the snout and the shift of the

661 naris were previously presumed to be peramorphic (Long \& McNamara, 1997), one can assume

662 on the basis of the current observations that the increase of gracility in the postorbital region of

663 derived sauropods may result from modular paedomorphosis. In this context, Salgado (1999) has 
664 hypothesized that the reduction of the supratemporal fenestra and fusion of the frontals in

665 diplodocoid sauropods is the result of a peramorphic heterochrony, while the loss of contact

666 between squamosal and quadratojugal could be paedomorphic. However, these character changes

667 are beyond the scope of the current study due to the lack of good skull material of juvenile

668 individuals of basal sauropods, and thus, need to be analysed in more detail in future studies after

669 the appropriate juvenile materials are discovered.

670

671 In contrast, the initial evolutionary changes in the skull shape of Theropoda were driven by

672 peramorphic events, as is observed in Coelophysis, the megalosaurid taxon and the hypothetical

673 ancestor of Neotheropoda in the regression analyses. These changes include the elongation of the

674 snout, increase in length of the antorbital fenestra, and trends to a relatively smaller orbit and

675 more robust post-rostral region. The basal ceratosaur Limusaurus has a rather small skull with a

676 short snout, enlarged subcircular orbit and gracile jugal and postorbital, so it is possible that the

677 more robust skull shape (oval orbit, massive jugal and postorbital) of large-bodied ceratosaurs

678 like Ceratosaurus and abelisaurids (e.g. Carnotaurus and Majungasaurus) could be the result of

679 a secondary peramorphosis as it was proposed for allosaurids and tyrannosaurids (e.g. Long \&

680 McNamara, 1997; Bhullar et al., 2012). However, due to the poor cranial knowledge and

681 fluctuating phylogenetic relationships of basal ceratosaurs from the Early and Middle Jurassic

682 (e.g. Pol and Rauhut, 2012; Tortosa et al., 2013), the early skull shape evolution of Ceratosauria

683 is not currently reproducible. In contrast, the skull of the hypothetical ancestor of Avetheropoda

684 is probably paedomorphic with respect to that of Orionides as shown in the regression analyses

685 (Fig. 4b, c). This trend might extend to the hypothetical ancestor of Coelurosauria,

686 Maniraptoriformes and Maniraptora, leading to a shorter, more tapering snout in lateral view, 
687 smaller antorbital fenestrae, enlarged subcircular orbits, and a more gracile postrostral region,

688 resembling the skull shape of the juvenile megalosaurid Sciurumimus. These findings may

689 indicate that the paedomorphic trend hypothesized for Eumaniraptora by Bhullar et al. (2012)

690 reaches back into the early evolution of Avetheropoda, and that basal coelurosaurs in fact

691 represent "miniaturized" tetanurans, conserving juvenile characters in adult individuals. A

692 similar trend is found for body size evolution in theropods, showing a successive decrease in

693 body size within Avetheropoda (Novas et al., 2012; Dececchi \& Larsson, 2013; Lee et al. 2014).

694 In contrast to this supposed early paedomorphic trend, the ancestral shape reconstruction reveals

695 that the skulls of allosauroids become secondarily more robust in relation to the hypothetical

696 ancestor of Avetheropoda, supporting cranial peramorphosis (see Canale et al., 2014). This

697 might also be the case for large-bodied tyrannosaurids (see Long \& McNamara, 1997; Bhullar et

698 al., 2012), although the current regression analyses could not find such a signal for both groups

699 (see below). Bhullar et al. (2012) suggested a multi-step progenetic paedomorphosis for skull

700 shape of Paraves and basal birds, with modular peramorphic trends related to beak formation,

701 and further peramorphic trends for secondarily large-bodied troodontids and dromaeosaurids.

702 These heterochronic changes were supported by trends regarding body size evolution (Turner et

703 al., 2007; Dececchi \& Larrson 2013; Lee et al. 2014) and growth patterns (Erickson et al., 2009)

704 found within Eumaniraptora. However, as it is the case for Sauropodomorpha, various trends

705 seen in skull shape evolution of theropods need to be verified in the future regarding possible

706 heterochrony on the basis of new material of both juvenile and adult specimens.

707

708 Functional and ecological implications 
709 The major differences in cranial shape found here clearly affect dietary preferences and

710 functional constraints. The robust morphology of the postorbital region and the oval orbit in

711 peramorphic skulls was previously discussed in relation to the generation of higher bite forces

712 (Henderson, 2002; Foth \& Rauhut, 2013a). However, these functional constraints go hand in

713 hand with a decrease in cranial disparity (Brusatte et al., 2012). Paedomorphic changes in the

714 orbital and postorbital regions were discussed in relation to visual elaboration and brain

715 enlargement (Bhullar et al., 2012), and may have played an important role in nocturnal activity

716 (Schmitz \& Motani, 2011) or the evolution of flight within Paraves (Balanoff et al., 2013). On

717 the other hand, large and circular orbits might simply correlate with reduced mechanical stresses

718 during biting (Henderson, 2002), which have been suggested to also influence size and shape of

719 the external naris, antorbital fenestra and infratemporal fenestra (Witmer, 1997; Witzel \&

720 Preuschoft, 2005; Witzel et al., 2011).

721

722 Both ontogenetic and phylogenetic variations in snout shape are likely related to dietary

723 preferences (Brusatte et al. 2012; Foth \& Rauhut 2013; see above), in which the shape of

724 premaxillae and maxillae partly determines the number and size of teeth (Henderson \&

725 Weishampel, 2002). Various examples of ontogenetic changes in the morphology and number of

726 teeth are documented in Saurischia, including the basal sauropodomorph Massospondylus,

727 coelophysoids (Colbert, 1989), basal tetanurans (Rauhut \& Fechner, 2005; Rauhut et al., 2012),

728 tyrannosaurids (Carr, 1999; Tsuihiji et al., 2011) and maniraptorans (Kundrát et al., 2008; Bever

$729 \&$ Norell, 2009). Based on these observations the evolutionary increase in the number of teeth

730 has been interpreted as peramorphic (Bever \& Norell, 2009). Tooth morphology, however, was

731 found to be a stronger indicator of diet than the shape of the snout itself (see Smith, 1993; 
732 Barrett, 2000; Barrett, Butler \& Nesbitt, 2011; Zanno \& Makovicky, 2011; Foth \& Rauhut,

733 2013a; Hendrickx \& Mateus, 2014). In this context, Rauhut et al. (2012) hypothesised based on

734 the similarities in the dentition of the juvenile megalosaurid Sciurumimus, adult compsognathids

735 (Stromer, 1934; Currie \& Chen, 2001; Peyer, 2006) and adult dromaeosaurids (Xu \& Wu, 2001;

736 Norell et al., 2006), that strongly recurved crowns with reduced or no mesial serrations may be

737 paedomorphic in the latter two taxa. This heterochrony probably results from the decrease of

738 body size observed in coelurosaurs (see above) and indicates an evolutionary shift in dietary

739 preferences to smaller prey (see also Zanno \& Makovicky, 2011).

740

741 Limitations

742 As is common in vertebrate paleontology, the current study has a limited sample size when

743 compared with extant neontological data sets (Brown \& Vavrek, 2015). The current results are

744 necessarily preliminary and must be viewed with caution especially because the sampling of

745 ontogenetic trajectories is considerably lower than the sampling of adult individuals.

746 Furthermore, trajectories are constructed using a single juvenile and adult specimen, with no

747 intermediate forms. A single multistage example for Tyrannosaurus presented by Bhullar et al.

748 (2012) has shown that during ontogeny the trajectory can change its direction considerably in a

749 multivariate PCA plot. This, in turn, has an important impact on the length of the trajectory and

750 its angle in relation to other trajectories. However, in regression analyses the difference with a

751 two-stage approach should be less substantial as multivariate shape information is transformed

752 into a single variable of shape for each stage with respect to its centroid size. The poor sample of

753 juveniles is a result of rarity and poor preservation in the fossil record, which seems to be due to

754 a number of factors, including preferred hunting of juveniles by predators (Hone \& Rauhut, 
755 2010) and a smaller likelihood of preservation, discovery, and collection because juveniles have

756 smaller body sizes and more fragile bones than adults (Brown et al., 2013). Thus, due to small

757 sample sizes, the statistical power of our analyses is generally low (see Cumming, Fidler \&

758 Vaux, 2007), limiting the explanatory power of our results. On the other hand, Brown \& Vavrek

759 (2015) recently demonstrated that the number of positive and negative allometries is

760 underestimated in smaller samples in both paleontological and neontological data sets.

761

762 Another issue affecting our results is that the juvenile individuals sampled here are all of

763 different early ontogenetic stages. The juvenile Massospondylus represents a composite of

764 several embryos close to hatching (Reisz et al., 2010); the megalosaurid taxon (i.e. Sciurumimus

765 albersdoerferi) is an early juvenile and its exact age could not be determined (Rauhut et al.,

766 2012); the age of the Coelophysis juvenile reconstructed is approximately one year old

767 (estimated by Colbert, 1990; Rinehart et al., 2009); the juvenile Tarbosaurus specimen is two to 768 three years old (Tsuihiji et al., 2011); and the juvenile Allosaurus is likely five to seven years old

769 (estimated based on Bybee, Lee \& Lamm, 2006; Loewen, 2009). Thus, the different ontogenetic

770 stages of the juvenile specimens and the small number of individuals for each ontogenetic series

771 most likely affected the length, but maybe also the slope of the calculated trajectories (and thus

772 the angles between the trajectories) (see Cardini \& Elton, 2007), including that of the

773 hypothetical ancestors. Furthermore, the uncertainty regarding the age of the specimens leads to

774 another weak point, as specimen age was not used to characterize the ontogenetic trajectories

775 (see above), which is a common problem in paleontology (e.g. McKinney, 1986; Klingenberg,

776 1998, Gould, 2000, Schoch, 2010; Bhullar et al., 2012). In consequence, the applied regression

777 analyses explored allometry and not heterochrony (see Klingenberg \& Spence, 1993; 
778 Klingenberg, 1998). The substitution of age by size, however, would imply similar growth

779 dynamics (i.e. proportionality between age and size) between ancestors and descendants, which

780 would consequently ignore heterochronic processes related to growth rates (i.e. progenesis and

781 acceleration). Although dinosaurs generally have higher growth rates compared to other non-

782 avian reptiles, histological studies reveal that growth rates are not identical (Erickson, Rogers \&

783 Yerby, 2001, 2004; Padian, de Ricqlès \& Horner, 2001; Sander et al., 2004; Erickson et al.,

784 2009; Grady et al., 2014; Werner \& Griebeler, 2014). Therefore, allometric patterns cannot be

785 used to infer heterochrony beyond paedomorphosis and peramorphosis as argued by Klingenberg

786 \& Spence (1993) and Klingenberg (1998). Taking the uncertainties related to the lengths and

787 slopes of the ontogenetic trajectories (due to incomplete ontogenetic series) and statistical

788 uncertainties (due to the small sample size) into account, the classifications of underlying

789 heterochronic processes would be misleading and probably erroneous.

790

791 In the current study, the interpretations of paedomorphosis and peramorphosis rely on the

792 significant shape differences between adult individuals of the ontogenetic trajectories expressed

793 by shape vectors in the regression analyses, for which the multivariate shape data were

794 transformed into a univariate shape variable. These differences are affected by type of shape

795 variable, but more importantly by the ancestral shapes, which in turn depend on the phylogenetic

796 relationships, the algorithm of time calibration (e.g. Bapst, 2014) and the method of

797 reconstruction (e.g. Martins, 1999; Webster \& Purvis, 2001). Thus, one has to be aware that the

798 application of different methods could result in slightly different ancestral shapes, affecting the

799 value of the shape variable. However, because the current sample covers all major linages of

800 basal saurischians except of crested taxa, which were found to impact the ancestral shape of the

801 skull roofs significantly (see Fig. S5, Table S6 in the Supplementary Information), the results of 
802 the ancestral reconstruction of adult individuals are viewed as valid. By using two different

803 shape variables (Regression score and Euclidean distance), it was possible to confirm significant

804 results through multiple methods.

805

806 The undefined trend found for Tarbosaurus in relation to the hypothetical ancestor of

807 Avetheropoda illustrates the limitations of our analyses. Our result is seemingly contradictory to

808 previous hypotheses and our ancestral shape reconstruction, which proposed peramorphosis as

809 the main driver of skull evolution in large-bodied tyrannosaurids (see above, Long \&

810 McNamara, 1997, Bhullar et al., 2012). As stated above, this result is most likely related to the

811 small sample size of ontogenetic trajectories as skulls with elongated and slender snouts are

812 considered to be peramorphic on the basis of the regression analyses. The inclusion of more

813 ontogenetic trajectories of large-bodied theropods would probably change this result in favour of

814 a trend towards a deeper snout. Furthermore, large-bodied tyrannosaurids like Tarbosaurus

815 descended from small-bodied coelurosaurian ancestors (Xu et al., 2004, 2006; Brusatte et al.,

816 2010; Rauhut et al., 2010; Benson et al., 2014), which means that the hypothetical inclusion of

817 an ontogenetic trajectory of a small-bodied basal coelurosaur (e.g. Compsognathus, Dilong,

818 Haplocheirus) and a respective hypothetical ancestor of Coelurosauria would probably change

819 the current results, leading to a secondary peramorphic trend in Late Cretaceous tyrannosaurids,

820 as suggested by previous authors. Thus, this result is very likely an artefact of incomplete

821 sampling. In this context, the limited number of ontogenetic series of basal sauropodomorphs

822 results only in a rough trend regarding the relationship between cranial ontogeny and evolution,

823 which cannot be extended to more general patterns in the skull shape evolution of basal

824 sauropods. 
825

\section{Conclusions}

827 The importance of heterochrony in non-avian dinosaur skull evolution is a relatively new

828 concept (see Long \& McNamara, 1997; Bhullar et al., 2012). This study quantitatively assesses

829 the impact of skull heterochrony across early saurischian evolution, allowing testing some of the

830 heterochronic trends proposed by Bhullar et al. (2012) and further highlights different vantages

831 of using morphometric data to elucidate heterochronic trends. We estimated hypothetical

832 ontogenetic trajectories in Saurischia, Neotheropoda, Orionides, and Avetheropoda using

833 ontogenetic trajectories of Massospondylus, Coelophysis, a megalosaurid taxon, Allosaurus and

834 Tarbosaurus. When compared using PCA, the ontogenetic trajectories of the terminal taxa show

835 great variation in length and direction, but still follow some very general patterns, including a

836 relatively elongated and dorsoventrally deeper preorbital region, decrease in orbit size and

837 increase in jugal robustness. General peramorphic skulls include more elongate and slender

838 snouts, elongate antorbital fenestrae, oval orbits, dorsoventrally shallower post-rostral regions,

839 and more massive maxillae, jugals, and postorbitals. Paedomorphic skulls show the opposite

840 features. The shape changes from the hypothetical ancestor of Saurischia to Massospondylus

841 were paedomorphic, as previously suggested by Bhullar et al. (2012). In contrast, skull evolution

842 of basal theropod taxa was probably affected by peramorphic trends. However, Avetheropoda

843 showed paedomorphic changes compared to Orionides. This might indicate that the

844 paedomorphic trend found for Eumaniraptora (see Bhullar et al., 2012) may reach back into the

845 early evolution of Avetheropoda. The hypothesized peramorphic evolution for skull shape of

846 allosaurids and tyrannosaurids could not be supported by the current study, but this probably

847 resulted from the small sample size of ontogenetic trajectories. Although our data showed 
848 minimal differences between our crested-taxa and non-crested taxa data sets and semi-landmark

849 and no semi-landmark data sets, it is important to fully evaluate all possible sources of trends,

850 especially when working with a small data set. As stated above, our study is hampered by the

851 preservation of the fossil record (mainly the poor sample of complete juvenile specimens) and

852 more finds will help to elucidate other evolutionary patterns related to heterochrony. With a

853 larger number of taxa comprising juvenile and adult stages it will be possible to further test

854 heterochronic hypotheses within Saurischia in more detail, and eliminate artefacts related to

855 sample size. Future studies may also examine ontogenetic histories of individual taxa that have

856 reasonably complete ontogenetic samples, such as Coelophysis, to evaluate which factors

857 (dietary preference, heterochrony, etc.) drive shape change in individual taxa. A larger number of

858 studies using geometric morphometrics for individual taxa as well as a more complete sampling

859 within Saurischia are necessary to more completely assess the importance of heterochronic

860 processes in both sauropodomorph and theropod skull shape. In addition, it would be of value to

861 explore modularity in saurischian skulls to project the investigation of heterochronic processes to

862 particular skull regions. In sum, this study demonstrates that heterochrony played an important

863 role in basal non-avian saurischian skull evolution building upon previous studies (Bhullar et al., 864 2012).

865

866 Acknowledgements:

867 We thank Oliver Rauhut (Bayerische Staatssammlung für Paläontologie und Geologie,

868 München), Miriam Zelditch (University of Michigan), Johannes Knebel (Ludwig Maximilians

869 University, München), Stefan Richter (University of Rostock), Walter Joyce and Eduardo

870 Ascarrunz (both University of Fribourg) for discussion, and Michel Laurin (Sorbonne 
871 Universités, Paris) for comments on an earlier version of the manuscript. We further thank

872 Matthew Lamanna (Carnegie Museum of Natural History, Pittsburgh), Alex Downs (Ruth Hall

873 Museum, Ghost Ranch), David Gillette (Museum of Northern Arizona, Flagstaff) and Xu Xing

874 (Institute of Vertebrate Paleontology and Paleoanthropology, Beijing) for access to collections.

875 This study benefitted especially from critical comments of Jesús Marugán-Lobón (Universidad

876 Autónoma de Madrid) and three anonymous reviewers.

877

878 References

879 Adams DC. 2014. A generalized K statistic for estimating phylogenetic signal from shape and 880 other high-dimensional multivariate data. Systematic Biology 63:685-697.

881 Adams DC, Otárola-Castillo E. 2013. geomorph: an R package for the collection and analysis of 882 geometricmorphometric shape data. Methods in Ecology and Evolution 4:393-399.

883 Adams DC, Rohlf FJ, Slice DE. 2004. Geometric morphometrics: ten years of progress

884 following the "revolution." Italian Journal of Zoology 71:5-16.

885 Adams DC, Rohlf FJ, Slice DE. 2013. A field comes of age: geometric morphometrics in the 886 21st century. Hystrix, the Italian Journal of Mammalogy 24:7-14.

887 Alberch P, Gould SJ, Oster GF, Wake DB. 1979. Size and shape in ontogeny and phylogeny. $888 \quad$ Paleobiology 5:296-317.

889 Balanoff AM, Rowe TB. 2007. Osteological description of an embryonic skeleton of the extinct 890 elephant bird, Aepyornis (Palaeognathae: Ratitae). Journal of Vertebrate Paleontology $891 \quad 27: 1-53$.

892 Balanoff AM, Bever GS, Rowe TB, Norell MA. 2013. Evolutionary origins of the avian brain. $893 \quad$ Nature 201:93-96. 
894 Bapst DW. 2014. Assessing the effect of time-scaling methods on phylogeny-based analyses in 895 the fossil record. Paleobiology 40:331-351.

896 Barrett PM. 2000. Prosauropod dinosaurs and iguanas: speculations on the diets of extinct 897 reptiles. In: Sues H-D ed. Evolution of herbivory in terrestrial vertebrates. Cambridge: 898 Cambridge University Press, 42-78.

899 Barrett PM, Rayfield EJ. 2006. Ecological and evolutionary implications of dinosaur feeding $900 \quad$ behaviour. Trends in Ecology and Evolution 21:217-224.

901 Barrett PM, Butler RJ, Nesbitt SJ. 2011. The roles of herbivory and omnivory in early dinosaur 902 evolution. Earth and Environmental Science Transactions of the Royal Society of 903 Edinburgh 101:383-396.

904 Benson RBJ, Campione NE, Carrano MT, Mannion PD, Sullivan C, Upchurch P, Evans SE. 2014. Rates of dinosaur body mass evolution indicate 170 million years of sustained ecological innovation on the avian stem lineage. PloS Biology 12:e1001853.

Berge C, Penin X. 2004. Ontogenetic allometry, heterochrony, and interspecific differences in 908 909 the skull of african apes, using tridimensional procrustes analysis. American Journal of Physical Anthropology 124:124-138.

Bever GS, Norell MA. 2009. The perinate skull of Byronosaurus (Troodontidae) with

911 observations on the cranial Ontogeny of paravian theropods. American Museum Novitates $912 \quad 3657: 1-51$.

913 Bhullar B-A, Marugán-Lobón J, Racimo F, Bever GS, Rowe TB, Norell MA, Abzhanov A. 914 2012. Birds have paedomorphic dinosaur skulls. Nature 487:223-226. 
915 Bhullar B-A. 2012. A phylogenetic approach to ontogeny and heterochrony in the fossil record: 916 cranial evolution and development in anguimorphan lizards (Reptilia: Squamata). Journal 917 of Experimental Zoology (MOL DEV EVOL) 318B:521-530.

918 Blomberg S, Garland TJ, AR I. 2003. Testing for phylogenetic signal in comparative data: 919 behavioral traits are more labile. Evolution 57:717-745.

920 Bonnan MF. 2004. Morphometric analysis of humerus and femur shape in Morrison sauropods: implications for functional morphology and paleobiology. Paleobiology 30:444-470.

922 Bookstein FL. 1991. Morphometric tools for landmark data. Cambridge: Cambridge University $923 \quad$ Press.

924 Bookstein FL, Schäfer K, Prossinger H, Seidler H, Fiedler M, Stringer C, Weber GW, Arsuaga J925 L, Slice DE, Rohlf FJ, Recheis W, Mariam AJ, Marcus LF. 1999. Comparing frontal 926 cranial profiles in archaic and modern Homo by morphometric analysis. The Anatomical $927 \quad$ Record 257:217-224.

Brown CM, Evans DC, Campione NE, O’ Brien LJ, Eberth DA. 2013. Evidence for taphonomic 929 size bias in the Dinosaur Park Formation (Campanian, Alberta), a model Mesozoic terrestrial alluvial - paralic system. Palaeogeography, Palaeoclimatology, Palaeoecology 372:108-122.

932 Brown CM, Vavrek MJ. 2015. Small sample sizes in the study of ontogenetic allometry; 933 implications for palaeobiology. PeerJ 3:e818.

934 Brusatte SL, Benton MJ, Ruta M, Lloyd GT. 2008. Superiority, competition, and opportunism in 935 the evolutionary radiation of dinosaurs. Science 321:1485-1488. 
936 Brusatte SL, Norell MA, Carr TD, Erickson GM, Hutchinson JR, Balanoff AM, Bever GS,

937 Choiniere JN, Makovicky PJ, Xu X. 2010. Tyrannosaur paleobiology: new research on 938 ancient exemplar organisms. Science 329:1481-1485.

939 Brusatte SL. 2011. Calculating the tempo of morphological evolution: rates of discrete character $940 \quad$ change in a phylogenetic context. In: Elewa AMT ed. Computational Paleontology.

$941 \quad$ Heidelberg: Springer, 53-74.

942 Brusatte SL, Carr TD, Norell MA. 2012. The osteology of Alioramus, a gracile and long-snouted 943 tyrannosaurid (Dinosauria: Theropoda) from the Late Cretaceous of Mongolia. Bulletin of 944 the American Museum of Natural History 366:1-197.

945 Brusatte SL, Montanari S, Sakamoto M, Harcourt-Smith WEH. 2012. The evolution of cranial 946 form and function in theropod dinosaurs: insight from geometric morphometrics. Journal 947 of Evolutionary Biology 25:365-377.

948 Butler RJ, Goswami A. 2008. Body size evolution in Mesozoic birds: little evidence for Cope's 949 rule. Journal of Evolutionary Biology 21:1673-1682.

Bybee PJ, Lee AH, Lamm E-T. 2006. Sizing the Jurassic theropod dinosaur Allosaurus:

951 assessing growth strategy and evolution of ontogenetic scaling of limbs. Journal of

952 Morphology 267:347-359.

953 Cabreira SF, Schultz CL, Bittencourt JS, Soares MB, Fortier DC, Silva LR, Langer MC. 2011.

954 New stem-sauropodomorph (Dinosauria, Saurischia) from the Triassic of Brazil. $955 \quad$ Naturwissenschaften 98:1035-1040.

956 Campione NE, Evans DC. 2011. Cranial growth and variation in edmontosaurs (Dinosauria: 957 Hadrosauridae): implications for Latest Cretaceous megaherbivore diversity in North $958 \quad$ America. PLoS ONE 6:e25186. 
959 Canale IC, Novas FE, Salgado L, Coria RA. 2014. Cranial ontogenetic variation in Mapusaurus 960 roseae (Dinosauria: Theropoda) and the probable role of heterochrony in

961 carcharodontosaurid evolution. Paläontolologische Zeitschrift:1-11.

962 Cardini A, Elton S. 2007. Sample size and sampling error in geometric morphometric studies of $963 \quad$ size and shape. Zoomorphology 126:121-134.

964 Carr TD. 1999. Craniofacial ontogeny in Tyrannosauridae (Dinosauria, Coelurosauria). Journal 965 of Vertebrate Paleontology 19:497-520.

966 Carr TD, Williamson TE. 2004. Diversity of late Maastrichtian Tyrannosauridae (Dinosauria:

967 Theropoda) from western North America. Zoological Journal of the Linnean Society $968 \quad 142: 479-523$.

969 Carrano MT, Benson RBJ, Sampson SD. 2012. The phylogeny of Tetanurae (Dinosauria:

970 Theropoda). Journal of Systematic Palaeontology 10:211-300.

971 Chinnery B. 2004. Morphometric analysis of evolutionary trends in the ceratopsian postcranial 972 skeleton. Journal of Vertebrate Paleontology 24:591-609.

973 Chinsamy A. 1993. Bone histology and growth trajectory of the prosauropod dinosaur 974 Massospondylus carinatus Owen. Modern Geology 18:319-329.

975 Colbert EH. 1989. The Triassic dinosaur Coelophysis. Museum of Northern Arizona Bulletin 57: $976 \quad 1-160$.

977 Colbert EH. 1990. Variation in Coelophysis bauri. In: Carpenter K, Currie PJ eds. Dinosaur 978 systematics: approaches and perspectives. Cambridge: Cambridge University Press, 8197990.

980 Collyer ML, Adams DC. 2007. Analysis of two-state multivariate phenotypic change in $981 \quad$ ecological studies. Ecology 88:683-692. 
982 Corti M. 1993. Geometric Morphometrics: an extension of the revolution. Trends in Ecology and 983 Evolution 8:302-303.

984 Cumming G, Fidler F, Vaux DL. 2007. Error bars in experimental biology. The Journal of Cell $985 \quad$ Biology 177:7-11.

986 Currie PJ, Chen P. 2001. Anatomy of Sinosauropteryx prima from Liaoning, northeastern China. 987 Canadian Journal of Earth Sciences 38:1705-1727.

988 Dal Sasso C, Maganuco S. 2011. Scipionyx samniticus (Theropoda: Compsognathidae) from the 989 Lower Cretaceous of Italy. Memorie della Società Italiana di Scienze Naturali e del Museo $990 \quad$ Civico di Storia Naturale di Milano 37:1-281.

991 Dececchi TA, Larsson HCE. 2013. Body and limb size dissociation at the origin of birds:

992 uncoupling allometric constraints across a macroevolutionary transition. Evolution

993 $67: 2741-2752$.

994 Drake AG. 2011. Dispelling dog dogma: an investigation of heterochrony in dogs using 3D 995 geometric morphometric analysis of skull shape. Evolution \& Development 13:204-213.

996

Drake AG, Klingenberg CP. 2008. The pace of morphological change: historical transformation 997 of skull shape in St Bernard dogs. Proceedings of the Royal Society B 275:71-76.

998

999

1000

1001

1002

1003

1004
gs. Proceedings of the Royal Society B 275:71-76.

Erickson GM, Rogers KC, Yerby SA. 2001. Dinosaur growth patterns and rapid avian growth rates. Nature 412:429-432.

Erickson GM, Makovicky PJ, Currie PJ, Norell MA, Yerby SA, Brochu CA. 2004. Gigantism and comparative life-history parameters of tyrannosaurid dinosaurs. Nature 430:772-775.

Erickson GM, Rauhut OWM, Zhou Z, Turner AH, Inouye BD, Hu D, Norell MA. 2009. Was dinosaurian physiology inherited by birds? Reconciling slow growth in Archaeopteryx. PLoS ONE 4:e7390. 
1005 Evans DC. 2010. Cranial anatomy and systematics of Hypacrosaurus altispinus, and a

1006 comparative analysis of skull growth in lambeosaurine hadrosaurids (Dinosauria:

1007 Ornithischia). Zoological Journal of the Linnean Society 159:398-434.

1008 Ezcurra MD. 2007. The cranial anatomy of the coelophysoid theropod Zupaysaurus rougieri

1009 (Upper Triassic, Argentina). Historical Biology 19:185-202.

1010 Ezcurra MD. 2012. Phylogenetic analysis of Late Triassic-Early Jurassic neotheropod dinosaurs:

1011 implications for the early theropod radiation. Journal of Vertebrate Paleontology, Program

$1012 \quad$ and Abstracts 32:91.

1013 Ezcurra MD, Butler RJ. 2015. Post-hatchling cranial ontogeny in the Early Triassic diapsid

1014 reptile Proterosuchus fergusi. Journal of Anatomy:1-16.

1015 Ezcurra MD, Novas FE. 2007. Phylogenetic relationships of the Triassic theropod Zupaysaurus

1016 rougieri from NWArgentina. Historical Biology 19:35-72.

1017 Fink WL. 1982. The conceptual relationship between ontogeny and phylogeny. Paleobiology

$1018 \quad 8: 254-264$.

1019 Forasiepi AM, Sánchez-Villagra MR. 2014. Heterochrony, dental ontogenetic diversity, and the

1020 circumvention of constraints in marsupial mammals and extinct relatives. Paleobiology

$1021 \quad 40: 222-237$.

1022 Foth C. 2013. Ontogenetic, macroevolutionary and morphofunctional patterns in archosaur

1023 skulls: a morphometric approach. Ludwig-Maximilians-Universität, München.

1024 Foth C, Rauhut OWM. 2013a. Macroevolutionary and morphofunctional patterns in theropod

1025 skulls: a morphometric approach. Acta Palaeontologica Polonica 58:1-16. 
1026 Foth C, Rauhut OWM. 2013b. The good, the bad, and the ugly: the influence of skull

1027 reconstructions and intraspecific variability in studies of cranial morphometrics in

1028 theropods and basal saurischians. PLOS ONE 8:e72007.

1029 Frederickson JA, Tumarkin-Deratzian AR. 2014. Craniofacial ontogeny in Centrosaurus $1030 \quad$ apertus. PeerJ 2:e252.

1031 Fritsch M, Bininda-Emonds ORP, Richter S. 2013. Unraveling the origin of Cladocera by 1032 identifying heterochrony in the developmental sequences of Branchiopoda. Frontiers in 1033 Zoology 10:35.

1034 Gerber S, Neige P, Eble GJ. 2007. Combining ontogenetic and evolutionary scales of 1035 morphological disparity: a study of early Jurassic ammonites. Evolution \& Development $1036 \quad 9: 472-482$.

1037 Gould SJ. 1977. Ontogeny and phylogeny. Cambridge: Harvard University Press.

1038 Gould SJ. 2000. Of coiled oysters and big brains: how to rescue the terminology of heterochrony, 1039 now gone astray. Evolution \& Development 2:241-248.

1040 Gow CE, Kitching JW, Raath MA. 1990. Skulls of the prosauropod dinosaur Massospondylus 1041 carinatus Owen in the collections of the Bernand Price Institute for Palaeontological 1042 Research. Palaeontologia Africana 27:45-58.

1043 Gower JC. 1975. Generalized Procrustes analysis. Psychometrika 40:33-51.

1044 Grady JM, Enquist BJ, Dettweiler-Robinson E, Wright NA, Smith FA. 2014. Evidence for 1045 mesothermy in dinosaurs. Science 344:1268-1272.

1046 Guenther MF. 2009. Influence of sequence heterochrony on hadrosaurid dinosaur postcranial 1047 development. The Anatomical Record 292:1427-1441. 
1048 Hammer O, Harper DAT, Ryan PD. 2001. PAST: paleontological statistics software package for 1049 education and data analysis. Palaeontologia Electronica 4:1-9.

1050 Hedrick BP, Dodson P. 2013. Lujiatun psittacosaurids: understanding individual and taphonomic $1051 \quad$ variation using 3D geometric morphometrics. PLoS ONE 8:e69265.

1052 Henderson DM. 2002. The eyes have it: the sizes, shapes, and orientations of theropod orbits as 1053 indicators of skull strength and bite force. Journal of Vertebrate Paleontology 22:766-778.

1054 Henderson DM, Weishampel DB. 2002. Convergent evolution of the maxilla-dental-complex 1055 among carnivorous archosaurs. Senckenbergiana lethaea 82:77-92.

1056 Hendrickx C, Mateus O. 2014. Abelisauridae (Dinosauria: Theropoda) from the Late Jurassic of 1057 Portugal and dentition-based phylogeny as a contribution for the identification of isolated $1058 \quad$ theropod teeth. Zootaxa 3759:1-74.

1059 Hennig W. 1966. Phylogenetic systematics. Urbana: University of Illinois Press.

1060 Hone DWE, Naish D, Cuthill I. 2012. Does mutual sexual selection explain the evolution of head 1061 crests in pterosaurs and dinosaurs? Lethaia 45:139-156.

1062 Hone DWE, Rauhut OWM. 2010. Feeding behaviour and bone utilization by theropod dinosaurs. $1063 \quad$ Lethaia 43:232-244.

1064 Horner JR, Goodwin MB. 2006. Major cranial changes during Triceratops ontogeny.

$1065 \quad$ Proceedings of the Royal Society B 273:2757-2761.

1066 Horner JR, Goodwin MB. 2009. Extreme cranial ontogeny in the Upper Cretaceous dinosaur 1067 Pachycephalosaurus. PLoS ONE 4:e7626.

1068 Hübner TR, Rauhut OWM. 2010. A juvenile skull of Dysalotosaurus lettowvorbecki

1069 (Ornithischia: Iguanodontia), and implications for cranial ontogeny, phylogeny, and 

396.

1072 Irmis RB. 2011. Evaluating hypotheses for the early diversification of dinosaurs. Earth and

1073 Environmental Science Transactions of the Royal Society of Edinburgh 101:397-426.

1074 Jackson DA. 1993. Stopping rules in principal components analysis: a comparison of heuristical 1075 and statistical approaches. Ecology 74:2204-2214.

1076 Klein N, Sander PM. 2007. Bone histology and growth of the prosauropod dinosaur

1077 Plateosaurus engelhardti von Mayer, 1837 from the Norian bonebeds of Trossingen

1078 (Germany) and Frick (Switzerland). Special Papers in Palaeontology 77:169-206.

1079 Klingenberg CP. 1998. Heterochrony and allometry: the analysis of evolutionary change in $1080 \quad$ ontogeny. Biological Reviews 73:79-123.

1081 Klingenberg CP. 2011. MorphoJ: an integrated software package for geometric morphometrics. 1082 Molecular Ecology Resources 11:353-357.

1083 Klingenberg CP, Gidaszewski NA. 2010. Testing and quantifying phylogenetic signals and 1084 homoplasy in morphometric data. Systematic Biology 59:245-261.

1085 Klingenberg CP, Spence JR. 1993. Heterochrony and allometry: lessons from the water strider 1086 genus Limnoporus. Evolution 47:1834-1853.

1087 Kundrát M, Cruickshank ARI, Manning TW, Nudds J. 2008. Embryos of therizinosauroid 1088 theropods from the Upper Cretaceous of China: diagnosis and analysis of ossification 1089 patterns. Acta Zoologica (Stockholm) 89:231-251.

1090 Langer MC, Ezcurra MD, Bittencourt JS, Novas FE. 2010. The origin and early evolution of 1091 dinosaurs. Biological Reviews 85:55-110. 
1092 Laurin M. 2004. The evolution of body size, Cope's rule and the origin of amniotes. Systematic 1093 Biology 53:594-622.

1094 Lautenschlager S. 2014. Morphological and functional diversity in therizinosaur claws and the 1095 implications for theropod claw evolution. Proceedings of the Royal Society B $1096 \quad 281: 20140497$.

1097 Lee MSY, Cau A, Naish D, Dyke GJ. 2014. Sustained miniaturization and anatomical innovation 1098 in the dinosaurian ancestors of birds. Science 345:562-566.

1099 Li D, Norell MA, Gao K, Smith ND, Makovicky PJ. 2010. A longirostrine tyrannosauroid from 1100 the Early Cretaceous of China. Proceedings of the Royal Society B 277:183-190.

1101 Lieberman DE, Carlo J, Ponc de Leóon M, Zollikofer CPE. 2007. A geometric morphometric 1102 analysis of heterochrony in the cranium of chimpanzees and bonobos. Journal of Human $1103 \quad$ Evolution 52:647-662.

1104 Loewen MA. 2009. Variation in the Late Jurassic theropod dinosaur Allosaurus: ontogenetic, 1105 functional, and taxonomic implications. University of Utah, Salt Lake City.

1106 Loewen MA, Irmis RB, Sertich JJW, Currie PJ, Sampson SD. 2013. Tyrant dinosaur evolution 1107 tracks the rise and fall of Late Cretaceous oceans. PLoS ONE 8:e79420.

1108 Long JA, McNamara KJ. 1997. Heterochrony: the key to dinosaur evolution. In: Wolberg DL, 1109 Stumps E, Rosenberg GD eds. Dinofest International. Philadelphia: Academy of Natural $1110 \quad$ Sciences, 113-123.

1111 Lü J, Yi L, Brusatte SL, Yang L, Li H, Chen L. 2014. A new clade of Asian Late Cretaceous 1112 long-snouted tyrannosaurids. Nature Communications 5:3788.

1113 Maddison WP. 1991. Squared-change parsimony reconstructions of ancestral states for 1114 continuous-valued characters on a phylogenetic tree. Systematic Zoology 40:304-314. 
1115 Maddison WP, Maddison DR. 2009. Mesquite: A modular system of evolutionary analysis.

$1116 \quad$ Version 2.72.

1117 Madsen JHJ, Welles SP. 2000. Ceratosaurus (Dinosauria, Theropoda), a revised osteology. Utah 1118 Geology Survey Miscellaneous Publication 00-2:1-80.

1119 Maiorini L, Farke AA, Kotsakis T, Piras P. 2015. Males resemble females: re-evaluating sexual 1120 dimorphism in Protoceratops andrewsi (Neoceratopsia, Protoceratopsidae). PLoS ONE $1121 \quad 10: \mathrm{e} 0126464$.

1122 Mallon JC, Holmes R, Eberth DA, Ryan MJ, Anderson JS. 2011. Variation in the skull of 1123 Anchiceratops (Dinosauria, Ceratopsidae) from the Horseshoe Canyon Formation (Upper 1124 Cretaceous) of Alberta. Journal of Vertebrate Paleontology 31:1047-1071.

1125 Mallon JC, Ryan MJ, Campbell JA. 2015. Skull ontogeny in Arrhinoceratops brachyops 1126 (Ornithischia: Ceratopsidae) and other horned dinosaurs. Zoological Journal of the 1127 Linnean Society:1-20.

1128 Martínez RN. 2009. Adeopapposaurus mognai, gen. et sp. nov. (Dinosauria: Sauropodomorpha), 1129 with comments on adaptations of basal Sauropodomorpha. Journal of Vertebrate

$1130 \quad$ Paleontology 29:142-164.

1131 Martínez RN, Sereno PC, Alcober OA, Colombi CE, Renne PR, Montañez IP, Currie BS. 2011.

1132 A basal dinosaur from the dawn of the dinosaur era in southwestern Pangaea. Science $1133 \quad 331: 206-210$.

1134 Martínez RN, Apaldetti C, Abelin D. 2013. Basal sauropodomorphs from the Ischigualasto

1135 Formation. Society of Vertebrate Paleontology Memoir 12:51-69.

1136 Martins EP. 1999. Estimation of ancestral states of continuous characters: a computer simulation 1137 study. Systematic Biology 48:642-650. 
1138 McKinney ML. 1986. Ecological causation of heterochrony: a test and implications for

1139 evolutionary theory. Paleobiology 12:282-289.

1140 McNamara KJ. 1982. Heterochrony and phylogenetic trends. Paleobiology 8:130-142.

1141 McNamara KJ, McKinney ML. 2005. Heterochrony, disparity, and macroevolution.

$1142 \quad$ Paleobiology 31:17-26.

1143 Mitteroecker P, Gunz P, Weber GW, Bookstein FL. 2004. Regional dissociated heterochrony in 1144 multivariate analysis. Annals of Anatomy 186:463-470.

1145 Mitteroecker P, Gunz P. 2009. Advances in geometric morphometrics. Evolutionary Biology $1146 \quad 36: 235-247$.

1147 Mitteroecker P, Gunz P, Bookstein FL. 2005. Heterochrony and geometric morphometrics: a

1148 comparison of cranial growth in Pan paniscus versus Pan troglodytes. Evolution \&

1149 Development 7:244-258.

1150 Molnar RE. 2005. Sexual selection and sexual dimorphism in theropods. In: Carpenter K ed. The

1151 carnivorous dinosaurs. Bloomington: Indiana University Press, 284-312.

1152 Norell MA, Clark JM, Turner AH, Makovicky PJ, Barsbold R, Rowe TB. 2006. A new

1153 droameosaurid theropod from Ukhaa Tolgod (Ömnögov, Mongolia). American Museum

$1154 \quad$ Novitates 3545:1-51.

1155 Novas FE, Ezcurra MD, Agnolín FL, Pol D, Ortíz R. 2012. New Patagonian Cretaceous theropod 1156 sheds light about the early radiation of Coelurosauria. Revista del Museo Argentino de 1157 Ciencias Naturales, nueva serie, 14:57-81.

1158 Padian K, Horner JR. 2011. The evolution of "bizarre structures" in dinosaurs: biomechanics, 1159 sexual selection, social selection or species recognition? Journal of Zoology 283:3-17. 
1160 Padian K, de Ricqlès AJ, Horner JR. 2001. Dinosaurian growth rates and bird origins. Nature $1161 \quad 412: 405-408$.

1162 Paradis E. 2012. Analysis of phylogenetics and evolution with R. New York: Springer.

1163 Paradis E, Claude J, Strimmer K. 2004. APE: analyses of phylogenetics and evolution in R 1164 language. Bioinformatics 20:289-290.

1165 Peyer K. 2006. A reconsideration of Compsognathus from the Upper Tithonian of Canjuers, 1166 southeastern France. Journal of Vertebrate Paleontology 26:879-896.

1167 Piras P, Salvi D, Ferrara G, Maiorino L, Delfino M, Pedde L, Kotsakis T. 2011. The role of post1168 natal ontogeny in the evolution of phenotypic diversity in Podarcis lizards. Journal of $1169 \quad$ Evolutionary Biology 24:2705-2720.

1170 Pol D, Rauhut OWM. 2012. A Middle Jurassic abelisaurid from Patagonia and the early 1171 diversification of theropod dinosaurs. Proceedings of the Royal Society B 279:3170-3175.

1172 Porfiri JD, Novas FE, Calvo JO, Agnolín FL, Ezcurra MD, Cerda IA. 2014. Juvenile specimen of 1173 Megaraptor (Dinosauria, Theropoda) sheds light about tyrannosauroid radiation.

$1174 \quad$ Cretaceous Research 51:35-55.

1175 Raff RA. 1996. The shape of life: genes, development, and the evolution of animal form.

1176 Chicago: University of Chicago Press.

1177 Rauhut OWM. 2003. The interrelationships and evolution of basal theropod dinosaurs. Special 1178 Papers in Palaeontology 69:1-213.

1179 Rauhut OWM, Fechner R, Remes K, Reis K. 2011. How to get big in the Mesozoic: the 1180 evolution of the sauropodomorph body plan. In: Klein N, Remes K, Gee CT, Sander PM 1181 eds. Biology of the sauropod dinosaurs: understanding the life of giants. Bloomington: 1182 Indiana University Press, 119-149. 
1183 Rauhut OWM, Foth C, Tischlinger H, Norell MA. 2012. Exceptionally preserved juvenile

1184 megalosauroid theropod dinosaur with filamentous integument from the Late Jurassic of

1185 Germany. Proceedings of the National Academy of Sciences 109:11746-11751.

1186 Rauhut OWM, Fechner R. 2005. Early development of the facial region in a non-avian theropod 1187 dinosaur. Proceedings of the Royal Society B 272:1179-1183.

1188 Rauhut OWM, Milner AC, Moore-Fay S. 2010. Cranial osteology and phylogenetic position of 1189 the theropod dinosaur Proceratosaurus bradleyi (Woodward, 1910) from the Middle $1190 \quad$ Jurassic of England. Zoological Journal of the Linnean Society 158:155-195.

1191 R-Development-Core-Team. 2011. R: a language and environment for statistical computing.

1192 Reilly SM, Wiley EO, Meinhardt DJ. 1997. An integrative approach to heterochrony: the 1193 distinction between interspecific and intraspecific phenomena. Biological Journal of the 1194 Linnean Society 60:119-143.

1195 Reisz RR, Evans DC, Sues H-D, Scott D. 2010. Embryonic skeletal anatomy of the 1196 sauropodomorph dinosaur Massospondylus from the Lower Jurassic of South Africa. 1197 Journal of Vertebrate Paleontology 30:1653-1665.

1198 Rinehart LF, Lucas SG, Heckert AB, Spielmann JA, Celeskey MD. 2009. The paleobiology of 1199 Coelophysis bauri (Cope) from the Upper Triassic (Apachean) Whitaker quarry, New 1200 Mexico, with detailed analysis of a single quarry block. New Mexico Museum of Natural $1201 \quad$ History and Science, Bulletin 45:1-260.

1202 Rohlf FJ. 2005. tpsDig, digitize landmarks and outlines, version 2.05.

1203 Rohlf FJ, Marcus LF. 1993. A revolution in morphometrics. Trends in Ecology and Evolution $1204 \quad 8: 129-132$. 
1205 Rohlf FJ, Slice DE. 1990. Extensions of the Procrustes method for the optimal superimposition $1206 \quad$ of landmarks. Systematic Zoology 39:40-59.

1207 Rowe TB. 1989. A new species of the theropod dinosaur Syntarsus from the Early Jurassic 1208 Kayenta Formation of Arizona. Journal of Vertebrate Paleontology 9:125-136.

1209 Sadleir RW, Barrett PM, Powell HP. 2008. The anatomy and systematics of Eustreptospondylus 1210 oxoniensis, a theropod dinosaur from the Middle Jurassic of Oxfordshire, England. 1211 Monograph of the Palaeontological Society 627:1-82.

1212 Sakamoto M. 2010. Jaw biomechanics and the evolution of biting performance in theropod 1213 dinosaurs. Proceedings of the Royal Society B 277:3327-3333.

1214 Salgado L. 1999. The macroevolution of the Diplodocimorpha (Dinosauria; Sauropoda): a 1215 developmental model. Ameghiniana 36:203-216.

1216 Sampson SD. 1999. Sex and destiny: the role of mating signals in speciation and 1217 macroevolution. Historical Biology 13:173-197.

1218 Sander PM, Klein N, Buffetaut E, Cuny G, Suteethorn V, Le Loeuff J. 2004. Adaptive radiation 1219 in sauropod dinosaurs: bone histology indicates rapid evolution of giant body size through $1220 \quad$ acceleration. Organisms, Diversity \& Evolution 4:165-173.

1221 Sander PM, Christian A, Clauss M, Fechner R, Gee CT, Griebeler EM, Gunga H-C, Hummel J, 1222 Mallison H, Perry SF, Preuschoft H, Rauhut OWM, Remes K, Tüttken T, Wings O, Witzel 1223 U. 2010. Biology of the sauropod dinosaurs: the evolution of gigantism. Biological $1224 \quad$ Reviews 86:117-155.

1225 Schmitz L, Motani R. 2011. Nocturnality in dinosaurs inferred from scleral ring and orbit 1226 morphology. Science 332:705-708. 
1227 Schoch RR. 2009. Life-cycle evolution as response to diverse lake habitats in Paleozoic 1228 amphibians. Evolution 63:2738-2749.

1229 Schoch RR. 2010. Heterochrony: the interplay between development and ecology exemplified by $1230 \quad$ a Paleozoic amphibian clade. Paleobiology 36:318-334.

1231 Schoch RR. 2014. Amphibian skull evolution: the developmental and functional context of 1232 simplification, bone loss and heterotopy. Journal of Experimental Zoology (MOL DEV $1233 \quad$ EVOL) 322B:619-630.

1234 Schwarz-Wings D, Böhm N. 2014. A morphometric approach to the specific separation of the 1235 humeri and femora of Dicraeosaurus from the Late Jurassic of Tendaguru/Tanzania. Acta 1236 Palaeontologica Polonica 59:81-98.

1237 Singleton M. 2002. Patterns of cranial shape variation in the Papionini (Primates: 1238 Cercopithecinae). Journal of Human Evolution 42:547-578.

1239 Slice DE. 2007. Geometric morphometrics. Annual Review of Anthropology 36:261-281.

1240 Smith KK. 1993. The form of the feeding apparatus in terrestrial vertebrates: studies of 1241 adaptation and constraint. In: Hanken J, Hall BK eds. The skull. Vol. 3. Patterns of 1242 structural and systematic diversity. Chicago: University of Chicago Press, 150-196.

1243 Smith ND, Makovicky PJ, Hammer WR, Currie PJ. 2007. Osteology of Cryolophosaurus ellioti 1244 (Dinosauria: Theropoda) from the Early Jurassic of Antarctica and implications for early 1245 theropod evolution. Zoological Journal of the Linnean Society 151:377-421.

1246 Stromer E. 1934. Die Zähne des Compsognathus und Bemerkungen über das Gebiß der 1247 Theropoda. Centralblatt für Mineralogie, Geologie und Paläontologie, B 1934:74-85. 
1248 Sues H-D, Nesbitt SJ, Berman DS, Henrici AC. 2011. A late-surviving basal theropod dinosaur 1249 from the latest Triassic of North America. Proceedings of the Royal Society B 278:3459$1250 \quad 3464$.

1251 Tallman M, Almécija S, Reber SL, Alba DM, Moyà-Solà S. 2013. The distal tibia of 1252 Hispanopithecus laietanus: more evidence for mosaic evolution in Miocene apes. Journal 1253 of Human Evolution 64:319-327.

1254 Therrien F, Henderson DM. 2007. My theropod is bigger than yours...or not: estimating body 1255 size from skull length in theropods. Journal of Vertebrate Paleontology 27:108-115.

1256 Tortosa T, Buffetaut E, Vialle N, Dutour Y, Turini E, Cheylan G. 2013. A new abelisaurid 1257 dinosaur from the Late Cretaceous of southern France: Palaeobiogeographical 1258 implications. Annales de Paléontologie 100:63-86.

1259 Tsuihiji T, Watabe M, Tsogtbaatar K, Tsubamoto T, Barsbold R, Suzuki S, Lee AH, Ridgely 1260 RC, Kawahara Y, Witmer LM. 2011. Cranial osteology of a juvenile specimens of 1261 Tarbosaurus bataar (Theropoda, Tyrannosauridae) from the Nemegt Formation (Upper 1262 Cretaceous) of Bugin Tsav, Mongolia. Journal of Vertebrate Paleontology 31:497-517. 1263 Turner AH, Pol D, Clarke JA, Erickson GM, Norell MA. 2007. A basal dromaeosaurid and size 1264 evolution preceding avian flight. Science 317:1378-1381.

1265 Turner AH, Makovicky PJ, Norell MA. 2012. A review of dromaeosaurid systematics and 1266 paravian phylogeny. Bulletin of the American Museum of Natural History 371:1-206.

1267 Tykoski RS. 1998. The osteology of Syntarsus kayentakatae and its implications for ceratosaurid 1268 phylogeny. Austin: The University of Texas, Austin.

1269 Tykoski RS, Rowe TB. 2004. Ceratosauria. In: Weishampel DB, Dodson P, Osmólska H eds. 1270 The Dinosauria. Berkeley: University of California Press, 47-70. 
1271 Webster AJ, Purvis A. 2002. Testing the accuracy of methods for reconstructing ancestral states

1272 of continuous characters. Proceedings of the Royal Society of London B 269:143-149.

1273 Werner J, Griebeler EM. 2014. Allometries of maximum growth rate versus body mass at

1274 maximum growth indicate that non-avian dinosaurs had growth rates typical of fast

1275 growing ectothermic sauropsids. PLoS ONE 9:e88834.

1276 Wilson JA, Sereno PC. 1998. Early evolution and higher-level phylogeny of sauropod dinosaurs.

1277 Society of Vertebrate Paleontology Memoir 5:1-68.

1278 Witmer LM. 1997. The evolution of the antorbital cavity of archosaurs: a study in soft-tissue

1279 reconstruction in the fossil record with an analysis of the function of pneumaticity. Society

$1280 \quad$ of Vertebrate Paleontology Memoir 3:1-73.

1281 Witzel U, Mannhardt J, Goessling R, Micheeli P, Preuschoft H. 2011. Finite element analyses

1282 and virtual syntheses of biological structures and their application to sauropod skulls. In:

1283 Klein N, Remes K, Gee CT, Sander PM eds. Biology of the sauropod dinosaurs:

1284 understanding the life of giants. Bloomington: Indiana University Press, 171-181.

1285 Witzel U, Preuschoft H. 2005. Finite-element model construction for the virtual synthesis of the

1286 skulls in vertebrates: case study of Diplodocus. The Anatomical Record 283A:391-401.

1287 Xu X, Norell MA, Kuang X, Wang X, Zhao Q, Jia C. 2004. Basal tyrannosauroids from China

1288 and evidence for protofeathers in tyrannosauroids. Nature 431:680-684.

1289 Xu X, Clark JM, Forster CA, Norell MA, Erickson GM, Eberth DA, Jia C, Zhao Q. 2006. A

1290 basal tyrannosauroid dinosaur from the Late Jurassic of China. Nature 439:715-718.

1291 Xu X, Wu X. 2001. Cranial morphology of Sinornithosaurus millenii Xu et al. 1999 (Dinosauria:

1292 Theropoda: Dromaeosauridae) from the Yixian Formation of Liaoning, China. Canadian

1293 Journal of Earth Sciences 38:1739-1752. 
1294 Young MT, Larvan MD. 2010. Macroevolutionary trends in the skull of sauropodomorph

1295 dinosaurs - the largest terrestrial animals to have ever lived. In: Elewa AMT ed.

1296 Morphometrics for non-morphometricans. Berlin: Springer, 259-269.

1297 Zanno LE, Makovicky PJ. 2011. Herbivorous ecomorphology and specialization patterns in 1298 theropod dinosaur evolution. Proceedings of the National Academy of Sciences 108:2321299237.

1300 Zelditch ML, Swiderski DL, Sheets HD. 2012. Geometric morphometrics for biologists: a 1301 primer. Amsterdam: Elsevier Academic Press.

1302 


\section{Figure and Table captions}

1306 Figure 1 Ontogenetic changes in the skull of saurischian dinosaurs. (a) General ontogenetic

1307 pattern in Saurischia exemplified for the basal theropod Coelophysis (adult specimen modified

1308 after Rauhut, 2003). (b-f) Specific ontogenetic changes in saurischian dinosaurs visualized as

1309 wireframes of Procrustes-fitted shapes. (b) Massospondylus. (c) Coelophysis. (d) Megalosaurid

1310 taxon. (e) Allosaurus. (f) Tarbosaurus. Grey dashed lines represent the juvenile stage and black

1311 solid lines represent the adult stage.

1312

1313 Figure 2 Principal component analysis of the main sample. (a) Ontogenetic trajectories of

1314 terminal taxa for PC 1 versus PC 2. (b) Ontogenetic trajectories of terminal taxa for PC 1 against

1315 PC 3. (c) Illustration of the main shape changes for the first three principal components.

1316 Theropod taxa are shown as black dots, while sauropodomorph taxa are shown as grey dots. The

1317 arrows illustrate the different ontogenetic trajectories, in which the arrowhead marks the position 1318 of the adult individual.

1320 Figure 3 Principal component analysis of ontogenetic trajectories. (a) Terminal and ancestral

1321 ontogenetic trajectories for PC 1 against PC 2. The arrows illustrate the different ontogenetic

1322 trajectories, in which the arrowhead marks the position of the adult individual and the base of the

1323 arrow indicates the juvenile individual. (b) Illustration of the main shape changes for the first two

1324 principal components.

1325 
1326 Figure 4 Centroid size regression analyses for the main sample. (a) Regression analysis of all

1327 terminal taxa including ontogenetic trajectories against log-transformed skull centroid size

1328 (LogCS) $(\mathrm{p}<0.0001)$. (b) Regression analysis of only terminal (solid arrows) and ancestral

1329 (dashed arrows) ontogenetic trajectories against log centroid size $(\mathrm{p}<0.0001)$ using the

1330 regression score as shape variable. (c) Equivalent regression analysis to (b) using the Euclidean

1331 distance as shape variable. Theropod taxa are shown as black dots, while sauropodomorph taxa

1332 are shown as grey dots. The arrows illustrate the different ontogenetic trajectories, in which the

1333 arrowhead marks the position of the adult individual and the base of the arrow indicates the

1334 juvenile individual.

1335

1336 Figure 5 Simplified phylogeny of Saurischia showing the main heterochronic trends of the

1337 skull. Peramorphosis is colored in green and paedomorphosis in yellow. Grey trends indicate

1338 uncertain shape trends. Shape of the hypothetical ancestors based on the continuous character

1339 mapping of the Procrustes-fitted shapes of the adult terminal taxa from the original data set. Blue

1340 skulls represent ancestral skull shapes for which ontogeny could not be analysed. The

1341 heterochronic trends found in the regression analyses are visualized by the color of the branches.

1342 Possible heterochronic trends related to the skull evolution of allosauroids and basal coelurosaurs

1343 (see discussion) are shown as dashed branches. 
1346 Table 1 Angles and length of terminal ontogenetic trajectories. Angles of ontogenetic

1347 trajectories against PC 1, pairwise angles between ontogenetic trajectories in the PC 1-PC 2 and

1348 PC 1-PC 3 morphospace and length of ontogenetic trajectories in the PC 1-PC 2 and PC 1-PC 3

1349 morphospace (Fig. 2a, b). Green fields mark pairwise angles in the PC 1-PC 2 morphospace and

1350 orange fields mark that of the PC 1-PC 3 morphospace. Angles, lengths and slopes of

1351 ontogenetic trajectories versus log-transformed centroid size (LogCS) (Fig. 4a).

1352

1353 Table 2 Angles and lengths of terminal and ancestral ontogenetic trajectories. Angles of

1354 ontogenetic trajectories against PC 1, pairwise angles between ontogenetic trajectories in the PC

1355 1-PC 2 morphospace and length of ontogenetic trajectories in the PC 1-PC 2 morphospace (Fig.

$13563 a)$.

1357

1358 Table 3 Angles and lengths of terminal and ancestral ontogenetic trajectories. Angles,

1359 lengths and slopes of ontogenetic trajectories from the regression of shape (Regression score, RS

1360 and Euclidean Distance, ED) versus log-transformed centroid size (LogCS) (Fig. 4b, c).

1361

1362 Table 4 Overview of heterochronies in saurischian skull shape. The differences of the

1363 regression scores $(\Delta \mathrm{RS})$ and the Euclidean distances $(\Delta \mathrm{ED})$ between ancestor-descendent

1364 relationships of adult individuals from the regression analysis (Fig. 4b, c) and the interpretation

1365 regarding heterochrony. $\triangle \mathrm{RS}$ and $\triangle \mathrm{ED}$ values in brackets mark insignificant trends. $\mathrm{NA}=$ not

1366 available. 


\section{Table $\mathbf{1}$ (on next page)}

Angles and length of terminal ontogenetic trajectories

Angles of ontogenetic trajectories against PC 1, pairwise angles between ontogenetic trajectories in the PC 1-PC 2 and PC 1-PC 3 morphospace and length of ontogenetic trajectories in the PC 1-PC 2 and PC 1-PC 3 morphospace (Fig. 2a, b). Green fields mark pairwise angles in the PC 1-PC 2 morphospace and orange fields mark that of the PC 1-PC 3 morphospace. Angles, lengths and slopes of ontogenetic trajectories versus log-transformed centroid size (LogCS) (Fig. 4a). 
1 Table 1 Angles and length of terminal ontogenetic trajectories. Angles of ontogenetic

2 trajectories against PC 1, pairwise angles between ontogenetic trajectories in the PC 1-PC 2 and

3 PC 1-PC 3 morphospace and length of ontogenetic trajectories in the PC 1-PC 2 and PC 1-PC 3

4 morphospace (Fig. 2a, b). Green fields mark pairwise angles in the PC 1-PC 2 morphospace and

5 orange fields mark that of the PC 1-PC 3 morphospace. Angles, lengths and slopes of

6 ontogenetic trajectories versus log-transformed centroid size (LogCS) (Fig. 4a).

\begin{tabular}{|l|c|c|c|c|c|}
\hline & Massospondylus & Coelophysis & Megalosaurid taxon & Allosaurus & Tarbosaurus \\
\hline Angle (PC 1-PC 2) & 85.6492 & 42.3458 & 83.3216 & 5.3228 & 3.7406 \\
\hline Length (PC 1-PC 2) & 0.1761 & 0.1174 & 0.1414 & 0.0332 & 0.0403 \\
\hline Angle (PC 1-PC 3) & 63.2316 & 10.1684 & 65.0464 & 18.5268 & 60.5157 \\
\hline Length (PC 1-PC 3) & 0.0297 & 0.0881 & 0.0390 & 0.0349 & 0.0818 \\
\hline & Massospondylus & Coelophysis & Megalosaurid taxon & Allosaurus & Tarbosaurus \\
\hline Massospondylus & 0 & 73.4000 & 128.2780 & 98.2417 & 177.2841 \\
\hline Coelophysis & 43.3033 & 0 & 54.8780 & 171.6416 & 109.3159 \\
\hline Megalosaurid taxon & 2.3276 & 40.9757 & 0 & 133.4803 & 54.4379 \\
\hline Allosaurus & 89.0280 & 132.3313 & 91.3556 & 0 & 79.0425 \\
\hline Tarbosaurus & 98.0914 & 141.3947 & 100.4190 & 9.0634 & 0 \\
\hline & Massospondylus & Coelophysis & Megalosaurid taxon & Allosaurus & Tarbosaurus \\
\hline Angle (LogCS) & 3.3947 & 4.8961 & 4.6105 & 0.2535 & 1.5851 \\
\hline Length (LogCS) & 2.2815 & 1.0636 & 1.8147 & 1.0657 & 1.4016 \\
\hline Slope (LogCS) & 0.0593 & 0.0857 & 0.0806 & 0.0044 & 0.0277 \\
\hline
\end{tabular}




\section{Table 2 (on next page)}

Angles and lengths of terminal and ancestral ontogenetic trajectories

Angles of ontogenetic trajectories against PC 1, pairwise angles between ontogenetic trajectories in the PC 1-PC 2 morphospace and length of ontogenetic trajectories in the PC 1PC 2 morphospace (Fig. 3a). 
1 Table 2 Angles and lengths of terminal and ancestral ontogenetic trajectories. Angles of ontogenetic trajectories against PC 1,

2 pairwise angles between ontogenetic trajectories in the PC 1-PC 2 morphospace and length of ontogenetic trajectories in the PC 1-PC

32 morphospace (Fig. 3a).

\begin{tabular}{|c|c|c|c|c|c|c|c|c|c|}
\hline & Saurischia & Massospondylus & Neotheropoda & Coelophysis & Orionides & Megalosaurid taxon & Avetheropoda & Allosaurus & Tarbosaurus \\
\hline Angle (PC 1-PC 2) & 29.5357 & 15.193 & 19.6691 & 4.0256 & 33.2773 & 35.5725 & 29.4664 & 65.5478 & 79.1993 \\
\hline Length (PC 1-PC 2) & 0.082 & 0.1372 & 0.1202 & 0.1162 & 0.0879 & 0.1571 & 0.0429 & 0.044 & 0.0735 \\
\hline & Saurischia & Massospondylus & Neotheropoda & Coelophysis & Orionides & Megalosaurid taxon & Avetheropoda & Allosaurus & Tarbosaurus \\
\hline Saurischia & 0 & & & & & & & & \\
\hline Massospondylus & 14.3427 & 0 & & & & & & & \\
\hline Neotheropoda & 9.8666 & 4.4761 & 0 & & & & & & \\
\hline Coelophysis & 25.5101 & 11.1674 & 15.6435 & 0 & & & & & \\
\hline Orionides & 3.7416 & 18.0843 & 13.6082 & 29.2517 & 0 & & & & \\
\hline Megalosaurid taxon & 6.0368 & 20.3795 & 15.9034 & 31.5469 & 2.2952 & 0 & & & \\
\hline Avetheropoda & 0.0693 & 14.2734 & 9.7973 & 25.4408 & 3.8109 & 6.1061 & 0 & & \\
\hline Allosaurus & 84.9165 & 99.2591 & 94.783 & 110.4266 & 81.1749 & 78.8797 & 84.9858 & 0 & \\
\hline Tarbosaurus & 71.265 & 85.6076 & 81.1315 & 96.7751 & 67.5234 & 65.2282 & 71.3343 & 13.6515 & 0 \\
\hline
\end{tabular}

4

5

6 


\section{Table 3(on next page)}

Angles and lengths of terminal and ancestral ontogenetic trajectories

Angles, lengths and slopes of ontogenetic trajectories from the regression of shape (Regression score, RS and Euclidean Distance, ED) versus log-transformed centroid size (LogCS) (Fig. 4b, c). 
1 Table 3 Angles and lengths of terminal and ancestral ontogenetic trajectories. Angles, lengths and slopes of ontogenetic

2 trajectories from the regression of shape (Regression score, RS and Euclidean Distance, ED) versus log-transformed centroid size

3 (LogCS) (Fig. 4b, c).

\begin{tabular}{|c|c|c|c|c|c|c|c|c|c|}
\hline Regression (RS) & Saurischia & Massospondylus & Neotheropoda & Coelophysis & Orionides & Megalosaurid taxon & Avetheropoda & Allosaurus & Tarbosaurus \\
\hline Angle (LogCS) & 4.3762 & 3.8814 & 5.1181 & 6.056 & 4.1743 & 5.029 & 3.0083 & 1.1845 & 0.7153 \\
\hline Length (LogCS) & 1.1084 & 2.2828 & 1.3988 & 1.0657 & 1.3267 & 1.8158 & 0.9628 & 1.0659 & 1.4011 \\
\hline Slope $(\log C S)$ & 0.0765 & 0.0678 & 0.0896 & 0.1061 & 0.0730 & 0.0880 & 0.0526 & 0.0207 & 0.0125 \\
\hline Regression (ED) & Saurischia & Massospondylus & Neotheropoda & Coelophysis & Orionides & Megalosaurid taxon & Avetheropoda & Allosaurus & Tarbosaurus \\
\hline Angle $(\log C S)$ & 3.4145 & 5.0440 & 4.0199 & 5.0905 & 2.7768 & 3.4451 & 1.7014 & -0.1758 & -0.1087 \\
\hline Length (LogCS) & 1.1071 & 2.2864 & 1.3967 & 1.0640 & 1.3248 & 1.8121 & 0.9619 & 1.0657 & 1.4010 \\
\hline Slope $(\log C S)$ & 0.0597 & 0.0883 & 0.0703 & 0.0891 & 0.0485 & 0.0602 & 0.0297 & -0.0031 & -0.0019 \\
\hline
\end{tabular}




\section{Table 4 (on next page)}

Overview of heterochronies in saurischian skull shape

The differences of the regression scores ( $\triangle \mathrm{RS}$ ) and the Euclidean distances ( $\triangle \mathrm{ED}$ ) between ancestor-descendent relationships of adult individuals from the regression analysis (Fig. $4 \mathrm{~b}$, c) and the interpretation regarding heterochrony. $\triangle R S$ and $\triangle E D$ values in brackets mark insignificant trends. NA = not available. 
1 Table 4 Overview of heterochronies in saurischian skull shape. The differences of the

2 regression scores $(\Delta \mathrm{RS})$ and the Euclidean distances $(\Delta \mathrm{ED})$ between ancestor-descendent

3 relationships of adult individuals from the regression analysis (Fig. 4b, c) and the interpretation

4 regarding heterochrony. $\Delta \mathrm{RS}$ and $\triangle \mathrm{ED}$ values in brackets mark insignificant trends. $\mathrm{NA}=$ not

5 available.

\begin{tabular}{|l|c|c|c|}
\hline & $\Delta R S$ & $\Delta E D$ & Heterochrony \\
\hline Saurischia-Massospondylus & -0.0262 & -0.0446 & Paedomorphosis \\
\hline Saurischia-Neotheropoda & 0.0629 & 0.0733 & Peramorphosis \\
\hline Neotheropoda-Coelophysis & 0.0140 & 0.0668 & Peramorphosis \\
\hline Neotheropoda-Orionides & 0.0146 & $\mathbf{( - 0 . 0 0 7 9 )}$ & NA \\
\hline Orionides-megalosaurid taxon & 0.0507 & 0.0497 & Peramorphosis \\
\hline Orionides-Avetheropoda & -0.0299 & -0.0256 & Paedomorphosis \\
\hline Avetheropoda-Allosaurus & 0.0153 & $\mathbf{( 0 . 0 0 6 6 )}$ & NA \\
\hline Avetheropoda-Tarbosaurus & -0.0145 & $\mathbf{( 0 . 0 0 1 5 )}$ & NA \\
\hline 95 \% CIs & 0.0078 & 0.0098 & \\
\hline Significance levels (p=0.05) & 0.0117 & 0.0147 & \\
\hline
\end{tabular}




\section{1}

Ontogenetic changes in the skull of saurischian dinosaurs

(a) General ontogenetic pattern in Saurischia exemplified for the basal theropod Coelophysis (adult specimen modified after Rauhut, 2003). (b-f) Specific ontogenetic changes in saurischian dinosaurs visualized as wireframes of Procrustes-fitted shapes. (b) Massospondylus. (c) Coelophysis. (d) Megalosaurid taxon. (e) Allosaurus. (f) Tarbosaurus. Grey dashed lines represent the juvenile stage and black solid lines represent the adult stage. 
(a)
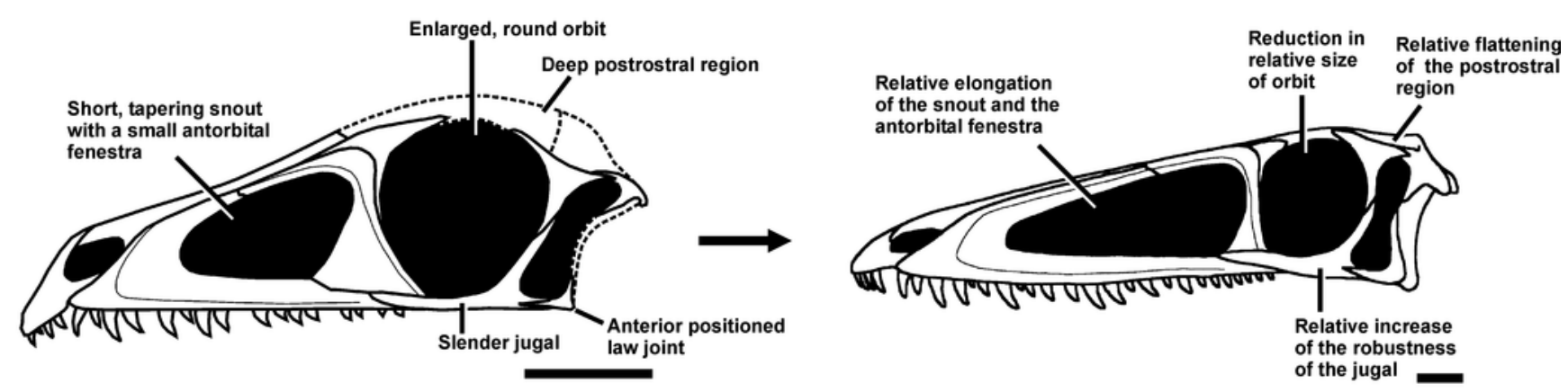

(b)

(c)
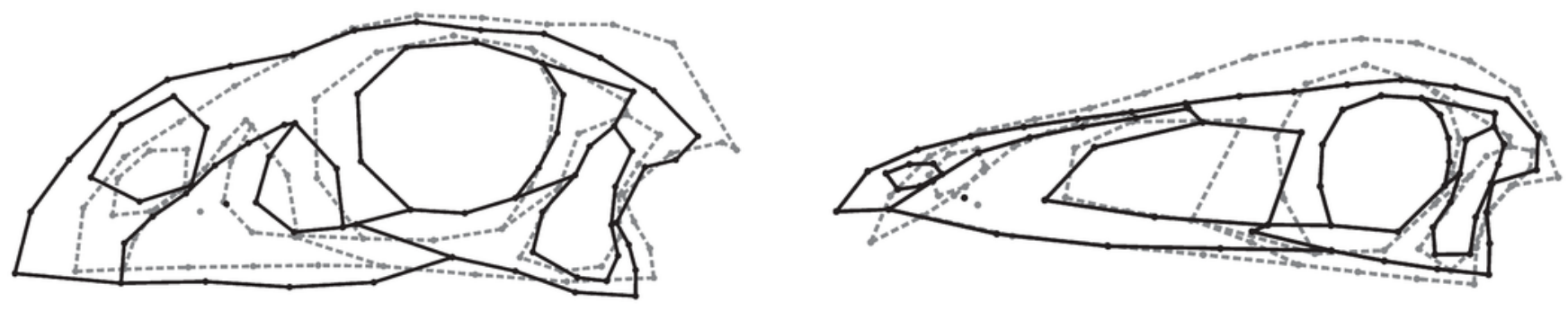

(d)

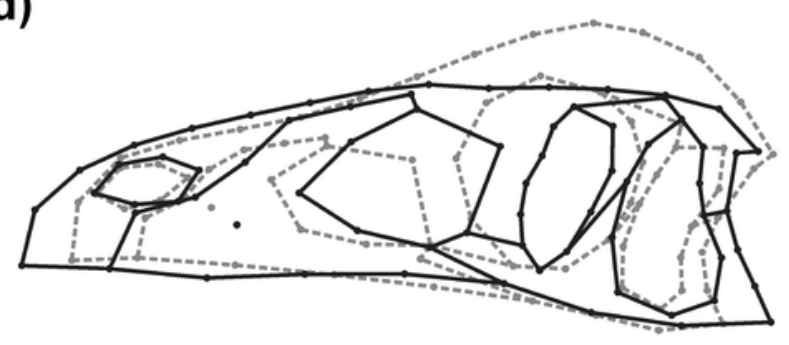

(e)

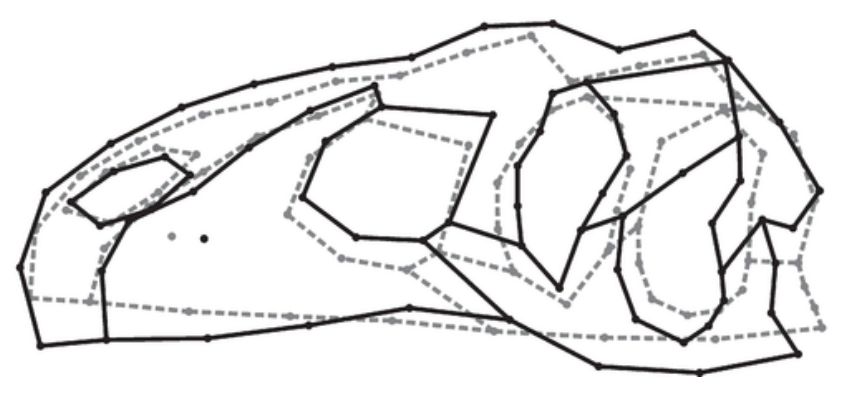

(f)

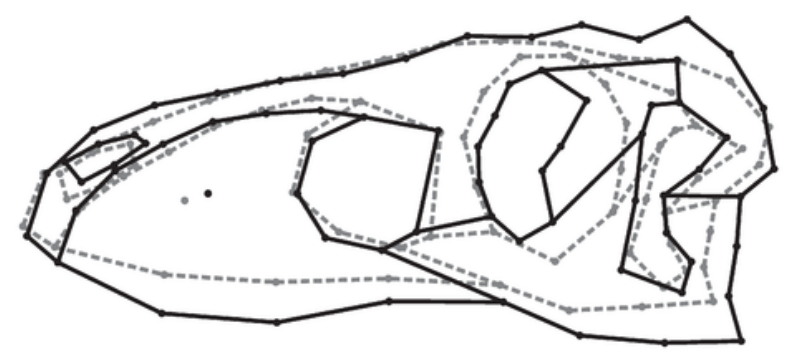


2

Principal component analysis of the main sample

(a) Ontogenetic trajectories of terminal taxa for PC 1 versus PC 2. (b) Ontogenetic trajectories of terminal taxa for PC 1 against PC 3. (c) Illustration of the main shape changes for the first three principal components. Theropod taxa are shown as black dots, while sauropodomorph taxa are shown as grey dots. The arrows illustrate the different ontogenetic trajectories, in which the arrowhead marks the position of the adult individual. 
(a)

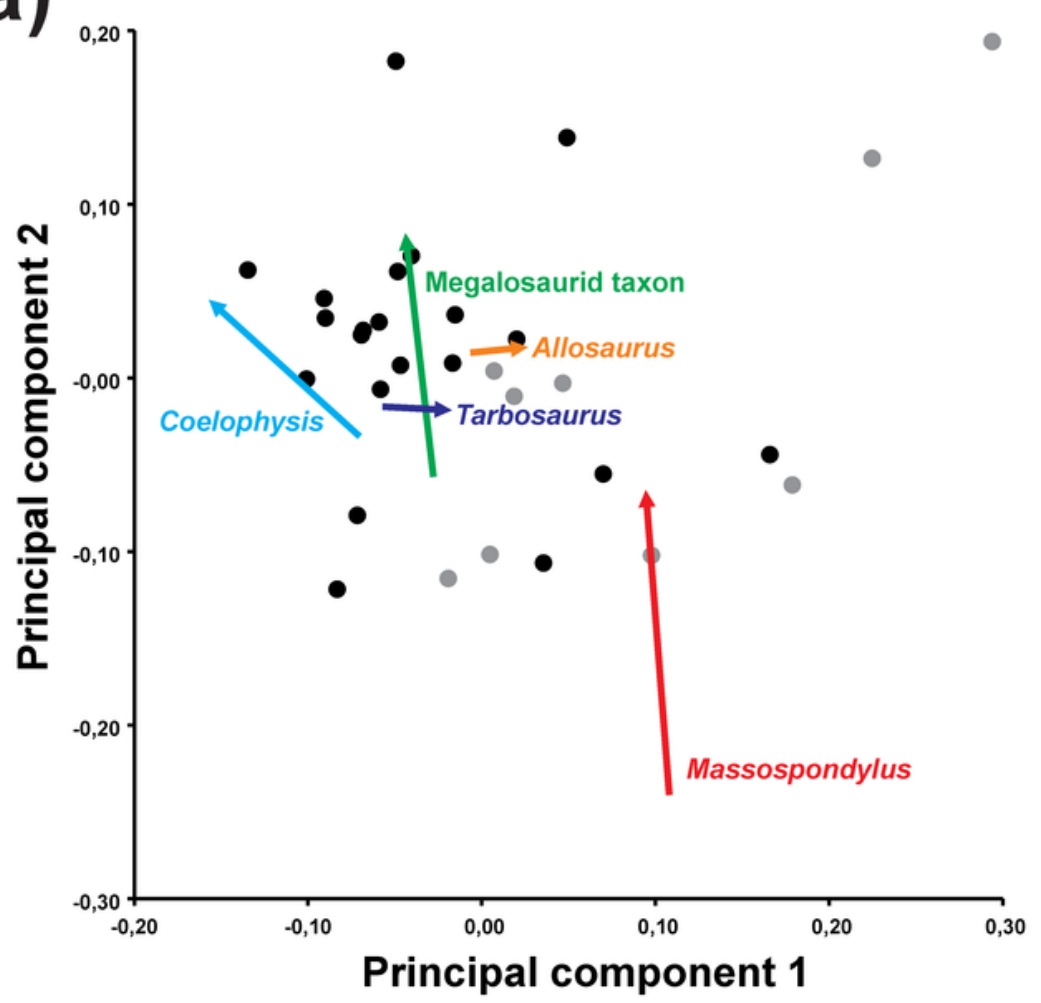

(b)

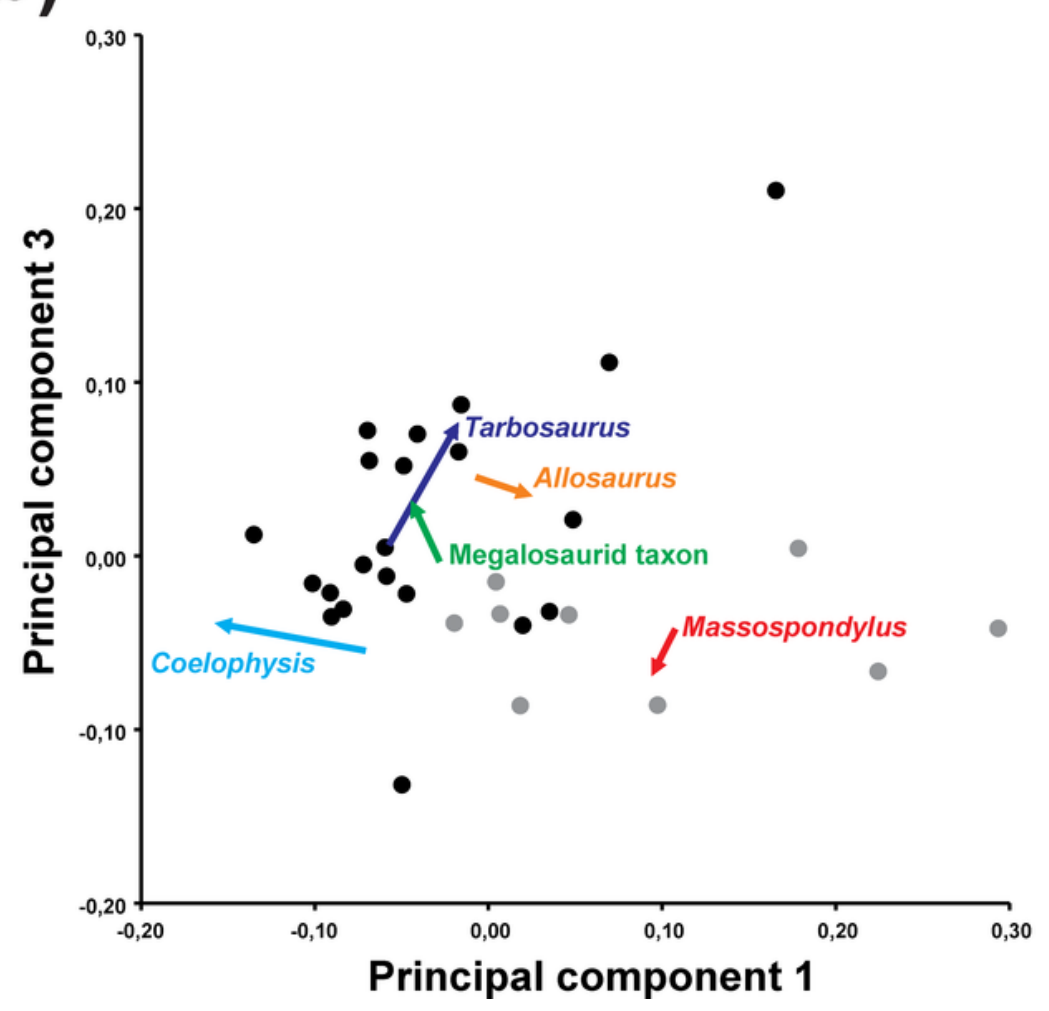

(c)
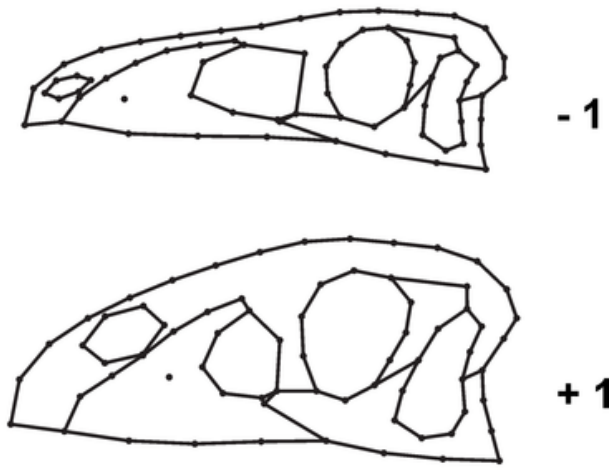

Principal component 1
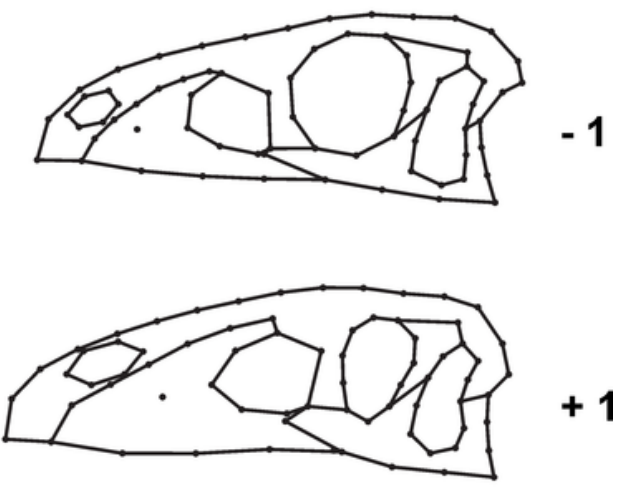

Principal component 2
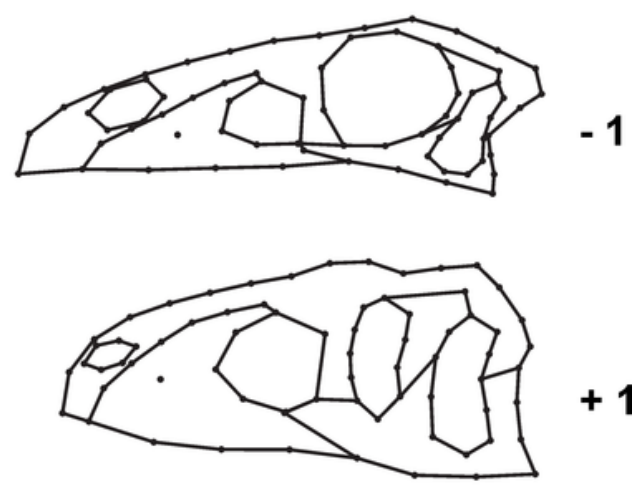

Principal component 3 
3

Principal component analysis of ontogenetic trajectories

(a) Terminal and ancestral ontogenetic trajectories for PC 1 against PC 2 . The arrows

illustrate the different ontogenetic trajectories, in which the arrowhead marks the position of the adult individual and the base of the arrow indicates the juvenile individual. (b) Illustration of the main shape changes for the first two principal components.

\section{(a)}

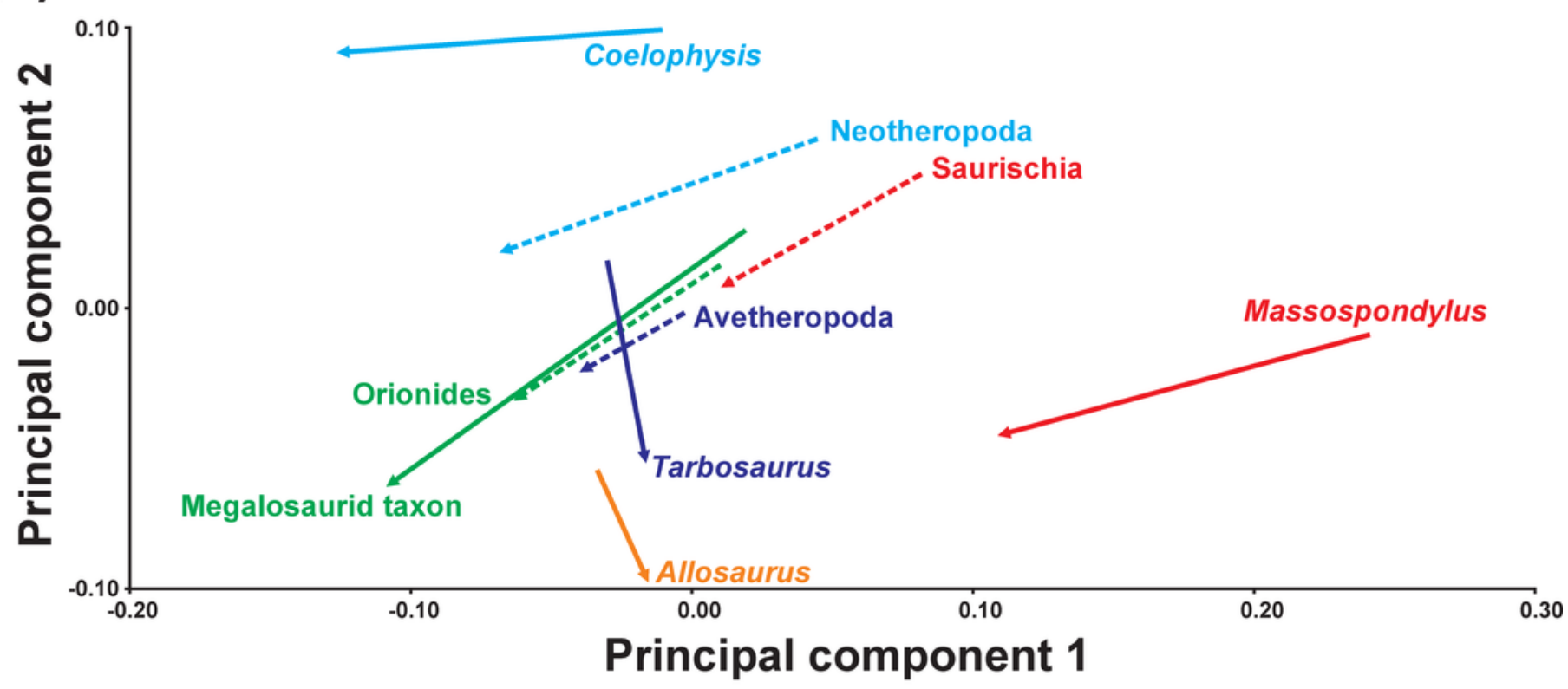

(b)
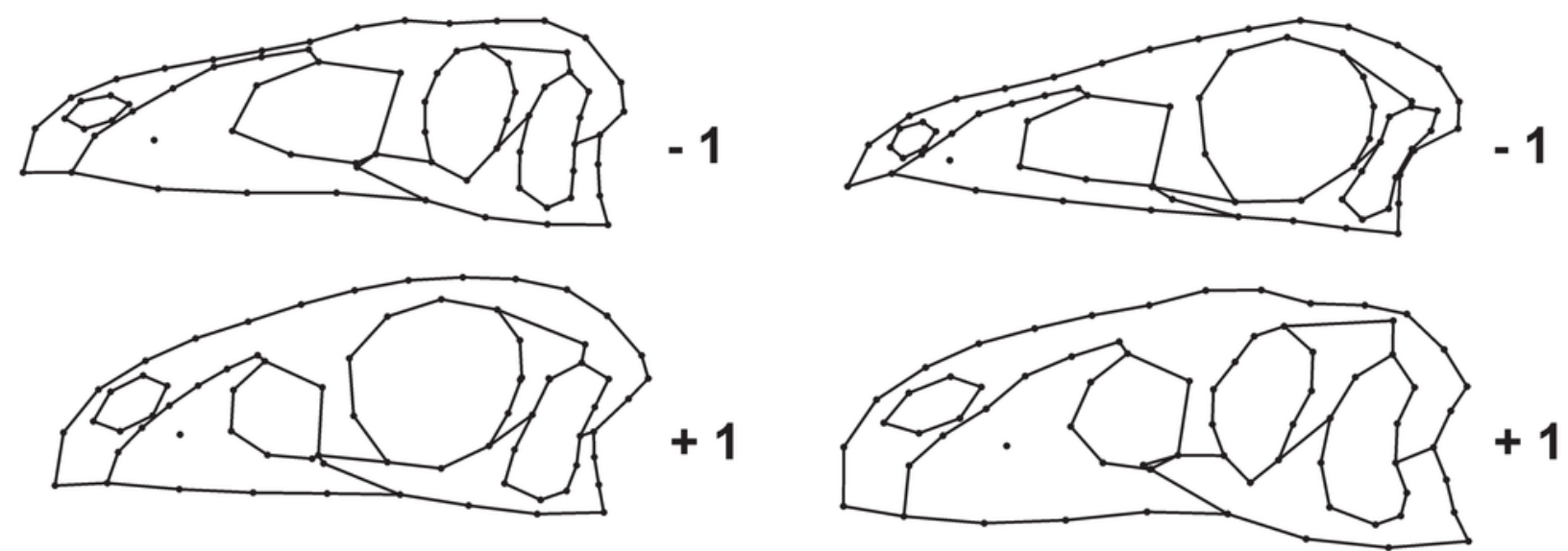

Principal component 1

Principal component 2 


\section{4}

Centroid size regression analyses for the main sample

(a) Regression analysis of all terminal taxa including ontogenetic trajectories against logtransformed skull centroid size (LogCS) $(p<0.0001)$. (b) Regression analysis of only terminal (solid arrows) and ancestral (dashed arrows) ontogenetic trajectories against log centroid size $(p<0.0001)$ using the regression score as shape variable. (c) Equivalent regression analysis to (b) using the Euclidean distance as shape variable. Theropod taxa are shown as black dots, while sauropodomorph taxa are shown as grey dots. The arrows illustrate the different ontogenetic trajectories, in which the arrowhead marks the position of the adult individual and the base of the arrow indicates the juvenile individual. 
(a)

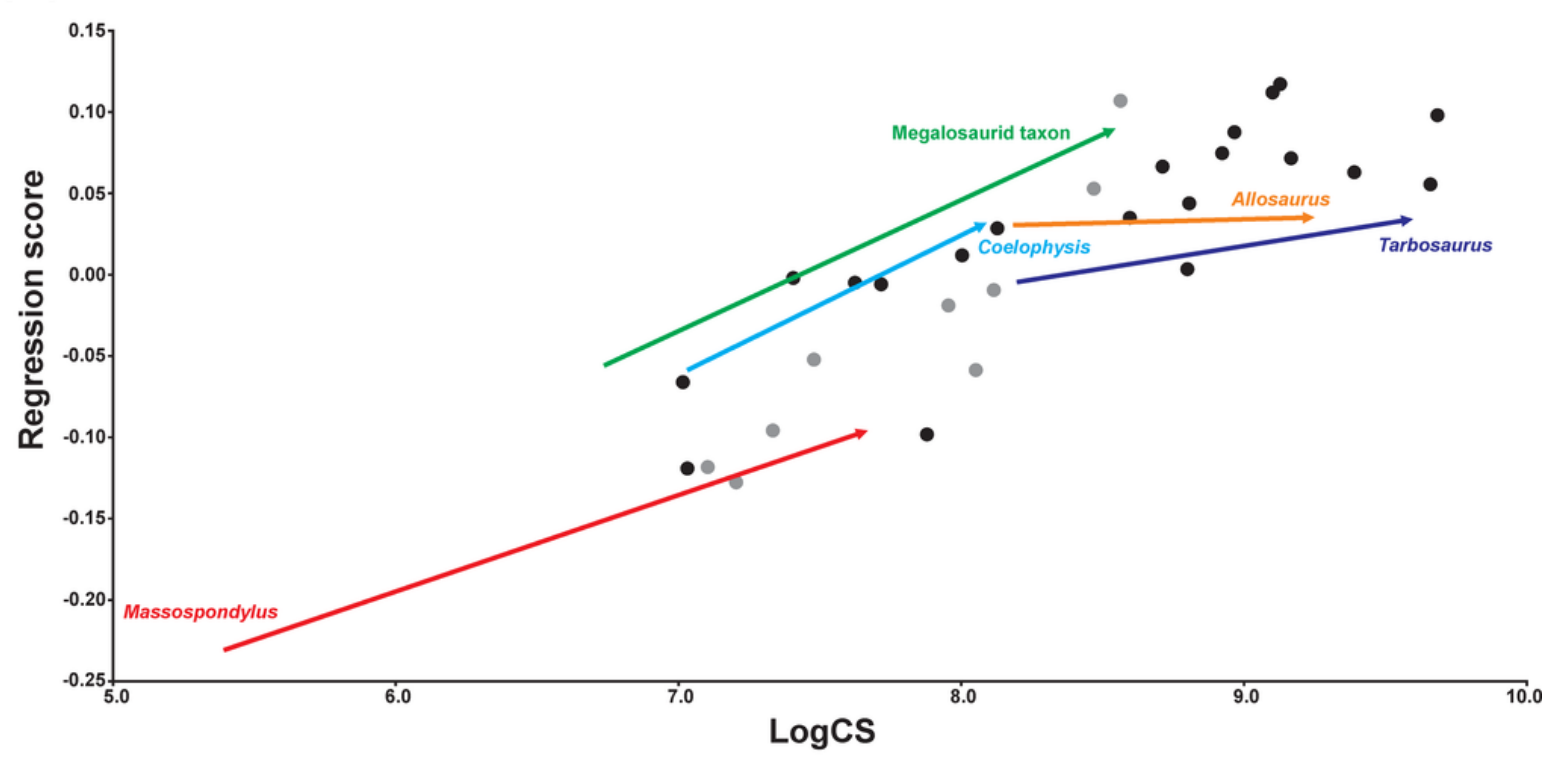

(b)

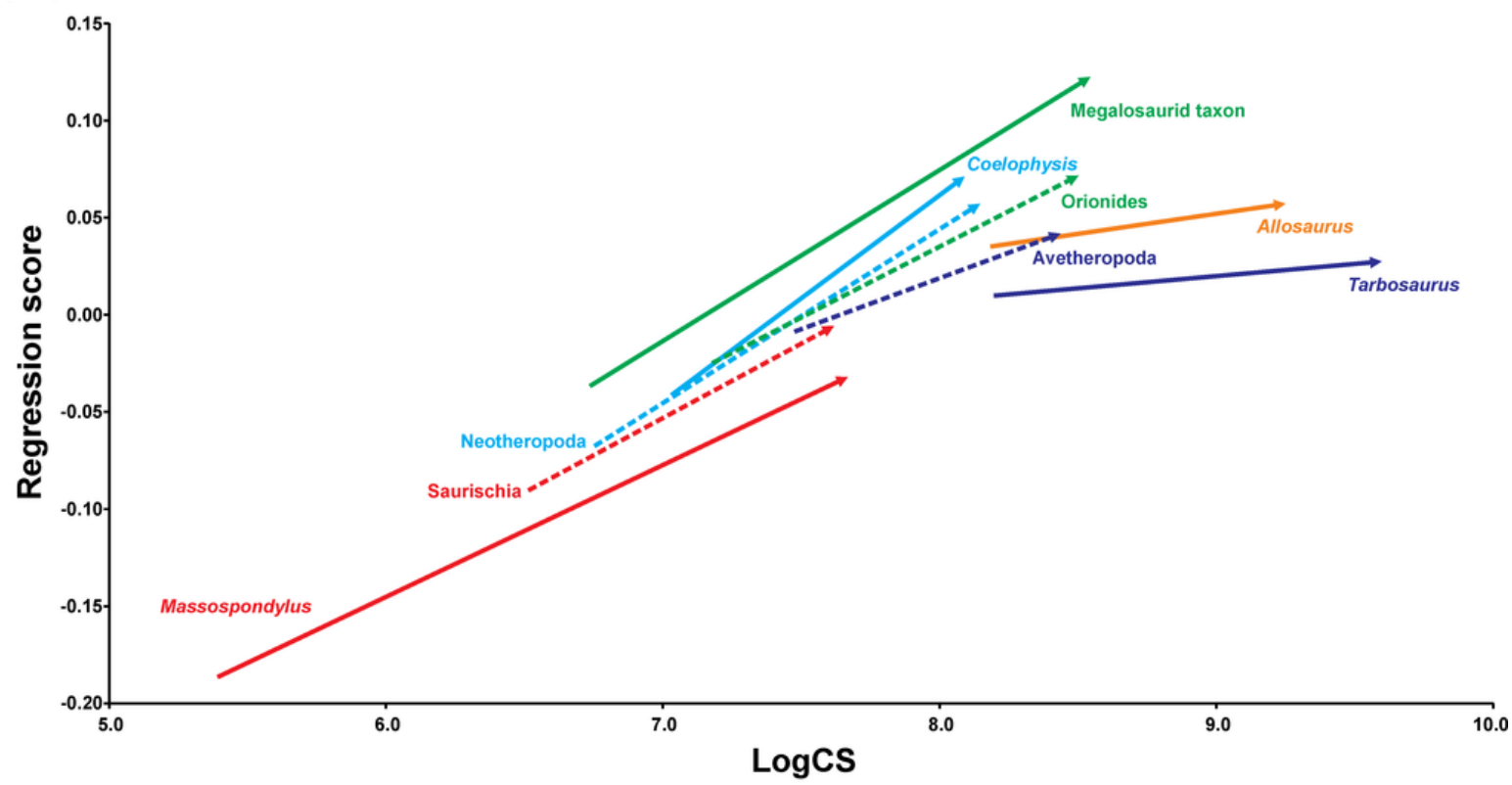

(c)

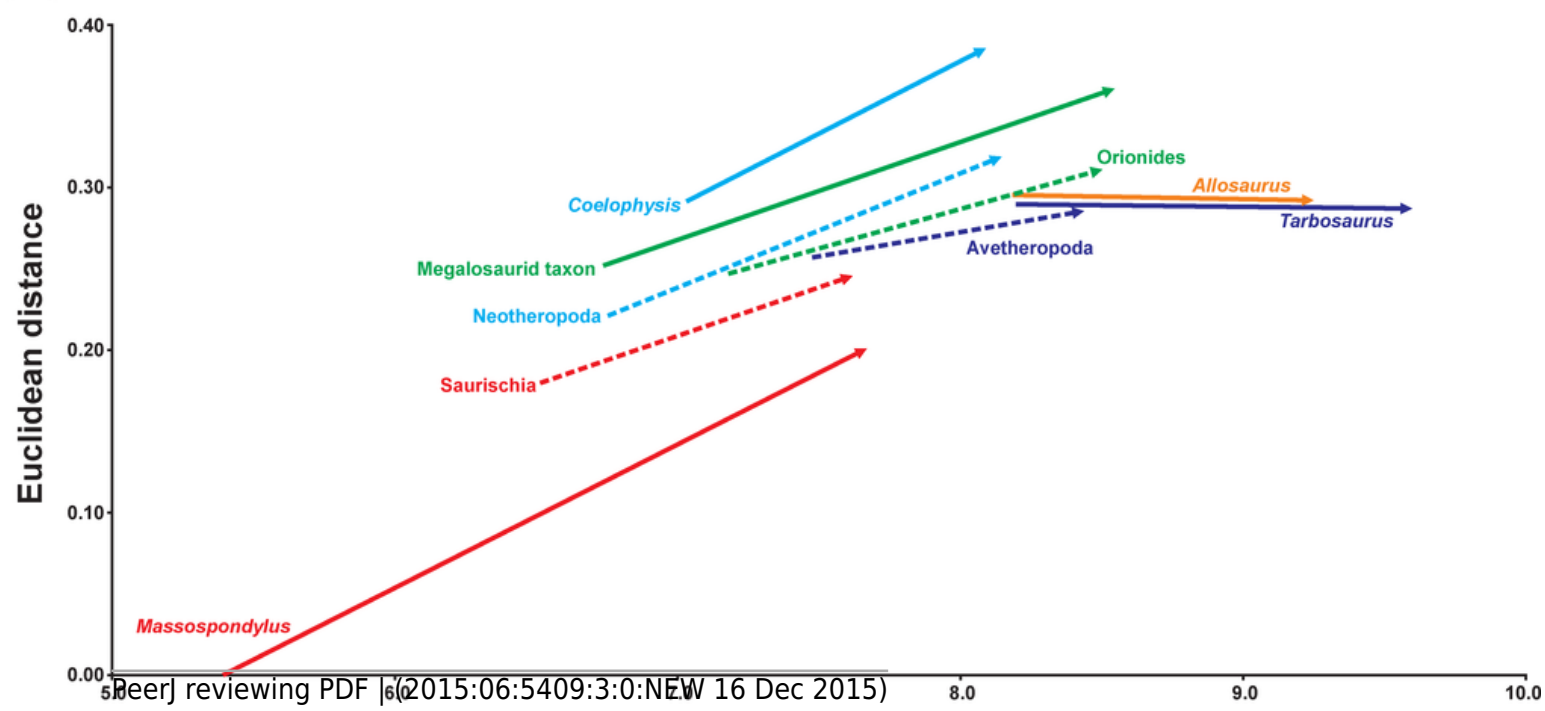
LogCS 


\section{5}

Simplified phylogeny of Saurischia showing the main heterochronic trends of the skull

Peramorphosis is colored in green and paedomorphosis in yellow. Grey trends indicate uncertain shape trends. Shape of the hypothetical ancestors based on the continuous character mapping of the Procrustes-fitted shapes of the adult terminal taxa from the original data set. Blue skulls represent ancestral skull shapes for which ontogeny could not be analysed. The heterochronic trends found in the regression analyses are visualized by the color of the branches. Possible heterochronic trends related to the skull evolution of allosauroids and basal coelurosaurs (see discussion) are shown as dashed branches. 


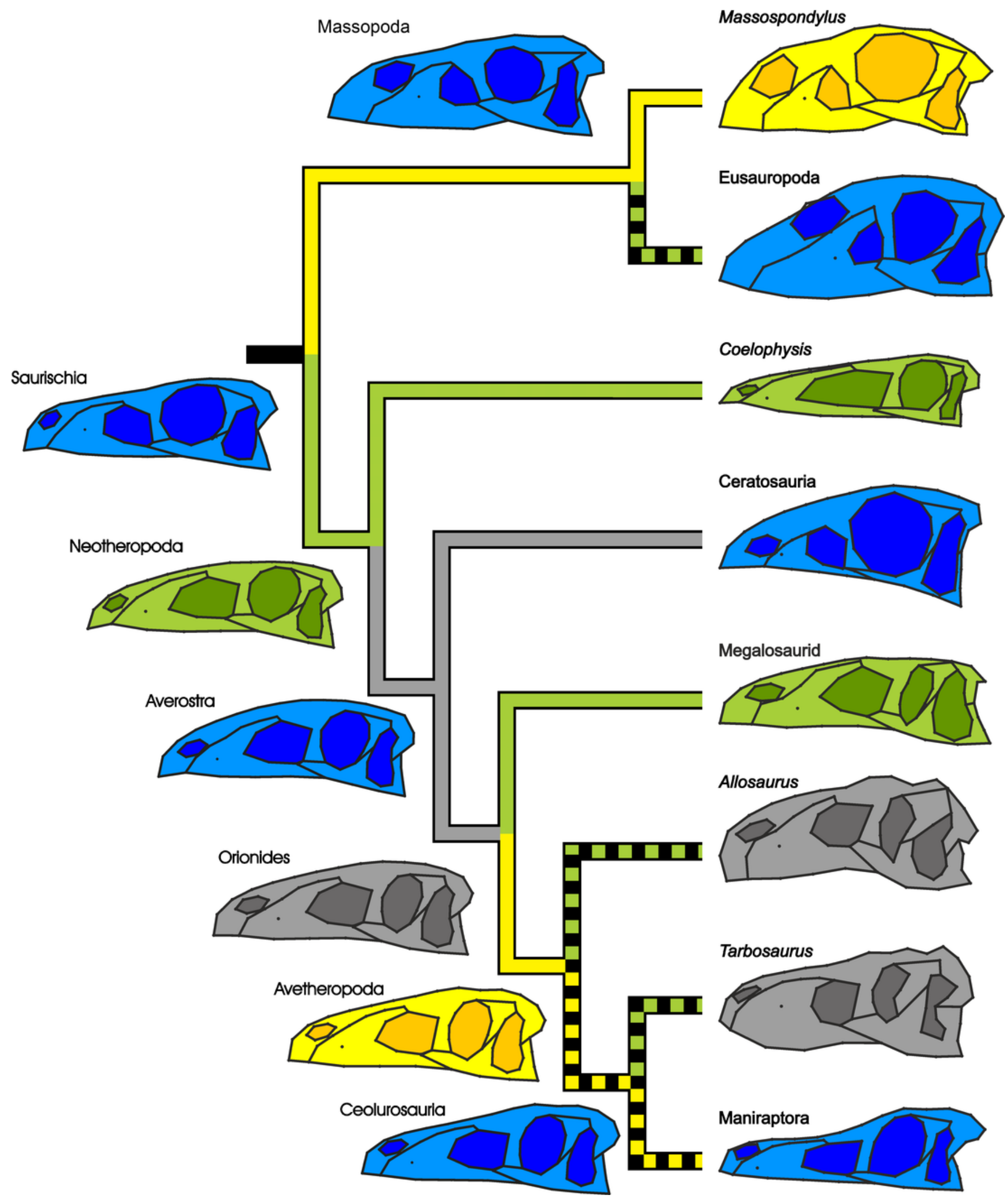

